\title{
Protein Kinase C Regulates Local Synthesis and Secretion of a Neuropeptide Required for Activity-Dependent Long-Term Synaptic Plasticity
}

\author{
Jiang-Yuan Hu, Yang Chen, and Samuel Schacher \\ Center for Neurobiology and Behavior, Columbia University College of Physicians and Surgeons, New York State Psychiatric Institute, New York, New York \\ 10032
}

\begin{abstract}
Long-term facilitation (LTF) of sensory neuron synapses in Aplysia is produced by either nonassociative or associative stimuli. Nonassociative LTF can be produced by five spaced applications of serotonin (5-HT) and requires a phosphoinosotide 3-kinase (PI3K)dependent and rapamycin-sensitive increase in the local synthesis of the sensory neuron neuropeptide sensorin and a protein kinase $\mathrm{A}$ (PKA)-dependent increase in the secretion of the newly synthesized sensorin. We report here that associative LTF produced by a single pairing of a brief tetanus with one application of 5-HT requires a rapid protein kinase C (PKC)-dependent and rapamycin-sensitive increase in local sensorin synthesis. This rapid increase in sensorin synthesis does not require PI3K activity or the presence of the sensory neuron cell body but does require the presence of the motor neuron. The secretion of newly synthesized sensorin by $2 \mathrm{~h}$ after stimulation requires both PKA and PKC activities to produce associative LTF because incubation with exogenous anti-sensorin antibody or the kinase inhibitors after tetanus plus 5-HT blocked LTF. The secreted sensorin leads to phosphorylation and translocation of p42/44 mitogenactivated protein kinase (MAPK) into the nuclei of the sensory neurons. Thus, different stimuli activating different signaling pathways converge by regulating the synthesis and release of a neuropeptide to produce long-term synaptic plasticity.
\end{abstract}

Key words: long-term facilitation; activity dependence; sensorin; local protein synthesis; protein kinase A; classical conditioning

\section{Introduction}

Long-term synaptic plasticity is an important cellular mechanism underlying learning and memory. Nonassociative and associative modifications of withdrawal reflexes in Aplysia are accompanied by changes in the properties of sensory neuron synapses (Kandel, 2001). Sensitization training or repeated applications of serotonin (5-HT) [nonassociative long-term facilitation (LTF)] produce increases in the following: (1) sensory neuron branches and varicosities, (2) sensory neuron varicosities with active zones for transmitter release, (3) transmitter release from existing presynaptic varicosities, (4) the size of the active zones, and (5) postsynaptic receptor responses (Bailey and Chen, 1983, 1988, 1989; Glanzman et al., 1990; Schacher et al., 1990; Bailey et al., 1992; Zhu et al., 1997; Kim et al., 2003; Roberts and Glanzman, 2003). Classical conditioning of withdrawal reflexes produced by pairing tactile stimulation with a sensitizing stimulus or temporal pairing of activity in sensory neurons with an application of 5-HT evoke parallel cellular changes in both presynaptic and postsyn-

Received May 21, 2007; revised June 25, 2007; accepted July 2, 2007.

This work was supported by National Institutes of Health Grants MH 60387 and NS 42159. Animals were provided by the National Center for Research Resources for Aplysia at the University of Florida (Miami, FL), which is supported by National Institutes of Health Grant RR-10294. We thank Dr. John Koester for his comments on previous drafts of this manuscript.

Correspondence should be addressed to Samuel Schacher, Center for Neurobiology and Behavior, Columbia University College of Physicians and Surgeons, New York State Psychiatric Institute, 1051 Riverside Drive, New York, NY10032.E-mail:sms2@columbia.edu.

DOI:10.1523/JNEUROSCI.2322-07.2007

Copyright $\odot 2007$ Society for Neuroscience $\quad$ 0270-6474/07/278927-13\$15.00/0 aptic neurons to produce associative LTF (Buonomano and Byrne, 1990: Sun and Schacher, 1998; Roberts and Glanzman, 2003). Do different stimuli activate the same or different signaling pathways to produce LTF?

5-HT can produce nonassociative LTF by activating several signaling pathways. LTF accompanied by structural plasticity requires new gene and protein expression (Bailey et al., 1992) mediated by the timely activation of phosphoinosotide 3-kinase (PI3K), protein kinase $\mathrm{A}(\mathrm{PKA})$, and mitogen-activated protein kinase (MAPK) in sensory neurons (Greenberg et al., 1987; Sweatt and Kandel, 1989; Nazif et al., 1991; Schacher et al., 1993; Martin et al., 1997; Michael et al., 1998; Muller and Carew, 1998; Purcell et al., 2003; Udo et al., 2005; Hu et al., 2006). The kinases phosphorylate both local cytoplasmic substrates (Schuster et al., 1985; Bailey et al., 1997; Martin et al., 1997; Michael et al., 1998; Angers et al., 2002; Liu et al., 2004) and transcription factors in the sensory neurons (Dash et al., 1990; Bacskai et al., 1993; Alberini et al., 1994; Martin et al., 1997; Bartsch et al., 1998; Yamamoto et al., 1999; Purcell et al., 2003). Protein kinase C (PKC) activity is enhanced by 5-HT (Sacktor and Schwartz, 1990; Sossin and Schwartz, 1992) and participates in both short- and intermediate-term facilitation (ITF) (Sugita et al., 1992, 1997; Wu et al., 1995; Sutton and Carew, 2000; Manseau et al., 2001; Sutton et al., 2004), but its role in nonassociative LTF is not clear (Sossin et al., 1994; Wu et al., 1995). Activation of MAPK in sensory neurons is mediated by 5 -HT-induced increases in the synthesis, secretion, and autocrine action of sensorin, the sensory 
neuron neuropeptide (Hu et al., 2004a, 2006) and other secreted factors that activate receptor tyrosine kinase (Purcell et al., 2003; Ormond et al., 2004; Sharma et al., 2006). PI3K- and rapamycinsensitive translation is required for the rapid increase in sensorin expression, whereas PKA activity is required for the subsequent release of the newly synthesized sensorin and for regulating sensorin signaling (Hu et al., 2006).

Does the same sequence of signal pathway activations and sensorin secretion contribute to associative LTF? The pairing of action potentials in sensory neurons with 5-HT leads to the entry of calcium that acts synergistically with 5-HT through calcium/ calmodulin-sensitive adenylyl cyclase to increase PKA activity sufficiently to produce LTF with a single pairing (Occor et al., 1985; Abrams et al., 1991, 1998; Sun and Schacher, 1998). Activity in the postsynaptic neuron and activation of PKC may also contribute to associative LTF (Lin and Glanzman, 1994; Murphy and Glanzman, 1997; Roberts and Glanzman, 2003; Sutton et al., 2004). We report here that sensorin synthesis and secretion are also required for associative LTF, but other signaling pathways than those required for nonassociative LTF are activated by the paired stimuli to regulate sensorin synthesis and secretion.

\section{Materials and Methods}

Cell culture and electrophysiology. Sensory neurons were isolated from pleural ganglia dissected from adult animals $(60-80 \mathrm{~g})$, and motor neuron L7s were isolated from juvenile abdominal ganglia ( $2 \mathrm{~g})$ and maintained in coculture for $5 \mathrm{~d}$ (Montarolo et al., 1986; Rayport and Schacher, 1986). The coculture contained one sensory neuron and one L7. Some dishes also contained sensory neurons plated alone. Standard electrophysiological techniques were used to record EPSP amplitudes evoked in L7 (Schacher et al., 1993; Hu et al., 2006). L7s were held at - $85 \mathrm{mV}$. EPSP amplitudes were recorded before and either 1 or $24 \mathrm{~h}$ after various treatments. Each sensory neuron was stimulated with a brief $(0.3-0.5 \mathrm{~ms})$ depolarizing pulse to evoke an action potential using an extracellular electrode placed near the cell body or axon stump of the sensory neuron. Cell bodies of some sensory neurons were removed as described previously (Hu et al., 2006). After $3 \mathrm{~h}$ of recovery, the cultures were treated as described below. Changes in EPSP amplitudes were measured by dividing the posttreatment EPSP amplitude by the pretreatment EPSP amplitude times $100 \%$. Some sensory neurons received a tetanus $(20 \mathrm{~Hz}$ for $2 \mathrm{~s})$ via the extracellular electrode plus a 5 min application of 5 -HT ( $50 \mu$ l of $50 \mu \mathrm{M}$ ) applied via micropipette placed near the SN-L7 pair (Eliot et al., 1994; Schacher et al., 1997; Sun and Schacher, 1998). Other sensory neurons received either a $5 \mathrm{~min}$ application of $5-\mathrm{HT}$ or tetanus alone. During the paired stimuli (tetanus plus 5-HT), the tetanus alone, or 5-HT alone, the motor neuron was held at its resting potential ( -50 to $-55 \mathrm{mV}$ ). Some cultures were exposed to five applications of 5-HT (5 min each at final concentration of $5 \mu \mathrm{M}$ ) applied at $20 \mathrm{~min}$ intervals. Cultures were rinsed after each application with $10 \mathrm{ml}$ of 1:1 mixture of L-15 medium and seawater. The protocol for nonassociative LTF lasted $90 \mathrm{~min}$.

Drug treatments. Cultures were incubated for $1-2 \mathrm{~h}$ with anti-sensorin antibody (Ab) or with protein A-purified preimmune serum $(400 \mathrm{ng} / \mathrm{ml})$ (Hu et al., 2004a) starting immediately after the washout of the application of 5-HT. The protein synthesis inhibitor rapamycin (50 nm; Sigma, St. Louis, MO), the PI3K inhibitor LY294002 [2-(4-morpholinyl)-8phenyl-1 $(4 H)$-benzopyran-4-one] (10 $\mu \mathrm{M}$; Calbiochem, La Jolla, CA), the PKA inhibitor KT5720 [(9S,10 S,12 R)-2,3,9,10,11,12-hexahydro-10hydroxy-9-methyl-1-oxo-9,12-epoxy- $1 \mathrm{H}$-diindolo[1,2,3-fg:3', $2^{\prime}, 1^{\prime}$ kl]pyrrolo[3,4-i] [1,6]benzodiazocine-10-carboxylic acid hexyl ester; 10 $\mu \mathrm{M}$; Calbiochem], or the PKC inhibitor chelyrethrine (10 $\mu \mathrm{M}$; Calbiochem) was added to some cultures 15 min before stimuli until $30 \mathrm{~min}$ after the stimuli. In other cultures LY294002, KT5720, or chelyrethrine was added for $90 \mathrm{~min}$ beginning at $30 \mathrm{~min}$ after the stimuli. The permeable peptide inhibitor S-Ht31 (10 $\mu \mathrm{M}$; Promega, Madison, WI) of type II PKA interaction with A-kinase anchoring proteins (AKAPs) or the mutated control peptide S-Ht31P (10 $\mu \mathrm{M}$; Promega) was added for $90 \mathrm{~min}$ beginning at $30 \mathrm{~min}$ after tetanus plus 5-HT (Liu et al., 2004; Hu et al., 2006). Cocultures or sensory neurons plated alone were exposed for 5 min to $100 \mathrm{~nm}$ of active phorbol [phorbol dibutyrate (PDBu) dissolved in DMSO; Sigma] or control inactive phorbol $(4 \alpha-\mathrm{PDBu}$ dissolved in DMSO; Sigma).

Immunocytochemistry. Immunocytochemistry was used to monitor the expression and distribution of sensorin throughout the sensory neurons and total p42/44 MAPK or phosphorylated p42/44 MAPK in the cell bodies of sensory neurons (Hu et al., 2004a, 2006). Cultures at various times after stimuli or after the application of control solutions were rinsed briefly in artificial seawater, fixed in $4 \%$ paraformaldehyde, and processed as described previously (Liu et al., 2003; Hu et al., 2004a). Cultures were exposed to rabbit polyclonal antibody specific for sensorin (1:1000) or total p42/44 MAPK and phospho-p42/44 MAPK (1:200; Cell Signaling Technology, Beverly, MA) diluted in $2 \%$ normal goat serum in $0.01 \mathrm{M}$ PBS with $0.3 \%$ Triton X-100 at $4^{\circ} \mathrm{C}$ for $24 \mathrm{~h}$. The incubated cultures were washed in $0.01 \mathrm{M} \mathrm{PBS}$ and incubated in FITC-conjugated goat anti-rabbit IgG (1:200; Sigma) at $4^{\circ} \mathrm{C}$ for $4 \mathrm{~h}$. After washing in $0.01 \mathrm{M}$ PBS, cultures were imaged directly with the appropriate filter set for detecting the fluorescent signal. The cultures were viewed with a Nikon (Tokyo, Japan) Diaphot microscope attached to a silicon-intensified target (SIT Dage 68; Dage-MTI, Michigan City, IN) video camera, the images were processed by a Dell computer (Dell Computer Company, Round Rock, TX), and images were captured and processed by the microcomputer-controlled imaging device (MCID) software package (Imaging Research, St. Catharines, Ontario, Canada). Illumination for detecting fluorescent signals was maintained at a constant setting for all experiments. Control experiments were performed to test the specificity of the primary antibody, including the substitution of normal rabbit serum for the primary antibody and omission of the primary antibody. All controls showed little immunocytochemical reaction.

Quantification and data analysis. All data are expressed as mean change (percentage) \pm SEM produced by the indicated treatments. The intensity of sensorin immunostaining was tested by measuring average fluorescent intensity in the sensory neuron cell body, in the entire main axon, and in varicosities contacting the major processes of L7 with the MCID (7.0) software package from Imaging Systems. Staining intensities for sensorin for the various experimental treatments were normalized to the intensities measured in each cellular compartment after control treatments (100\%). The overall staining intensity of total MAPK or phosphorylated MAPK immunofluorescence was determined by averaging intensity for the cell body (cytoplasm plus nucleus). Staining in the nucleus or cytoplasm was determined by measuring average intensity over that area. ANOVA and Scheffé's $F$ test were used to gauge significant differences between treatments.

\section{Results}

\section{Tetanus plus 5-HT induces a rapid increase in sensorin expression and secretion to produce associative LTF}

Nonassociative LTF produced by five applications of 5-HT requires the rapid increase in sensorin synthesis and the release of the newly synthesized sensorin (Hu et al., 2004a, 2006). We therefore tested whether a single pairing of tetanus plus 5-HT, known to produce associative LTF, evokes rapid changes in sensorin expression in the cell body, axon, and varicosities of sensory neurons.

Tetanus or tetanus plus 5-HT, but not 5-HT alone, produced a significant increase in the expression of sensorin, especially in the axon and distal varicosities of the sensory neurons (Fig. $1 \mathrm{~A}-$ $C)$. The increase in staining for sensorin was concentrated in punctate granules along the sensory neuron axon and in the distal sensory neuron varicosities contacting L7 (Fig. $1 A, B$ ). Compared with controls ( $n=15$ cultures; normalized to $100 \%$ ), staining for sensorin in the axon at $0.5 \mathrm{~h}$ increased by 2.5 -fold $(251 \pm 25 \%)$ after tetanus $(n=6)$ or by nearly threefold $(293 \pm 19 \%)$ after tetanus plus 5 -HT $(n=11)$. Sensorin staining in the varicosities at $0.5 \mathrm{~h}$ increased by nearly 2.5 -fold after tetanus $(230 \pm 13 \%)$ or 

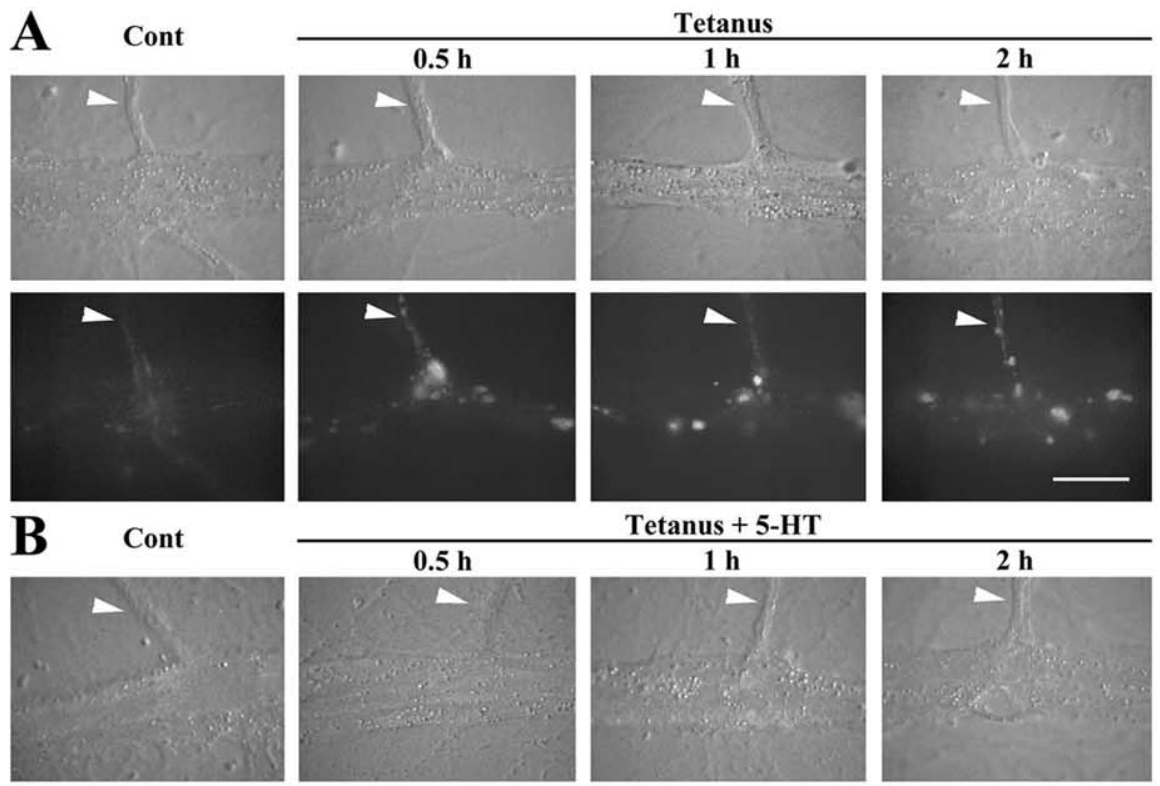

Tetanus + 5-HT
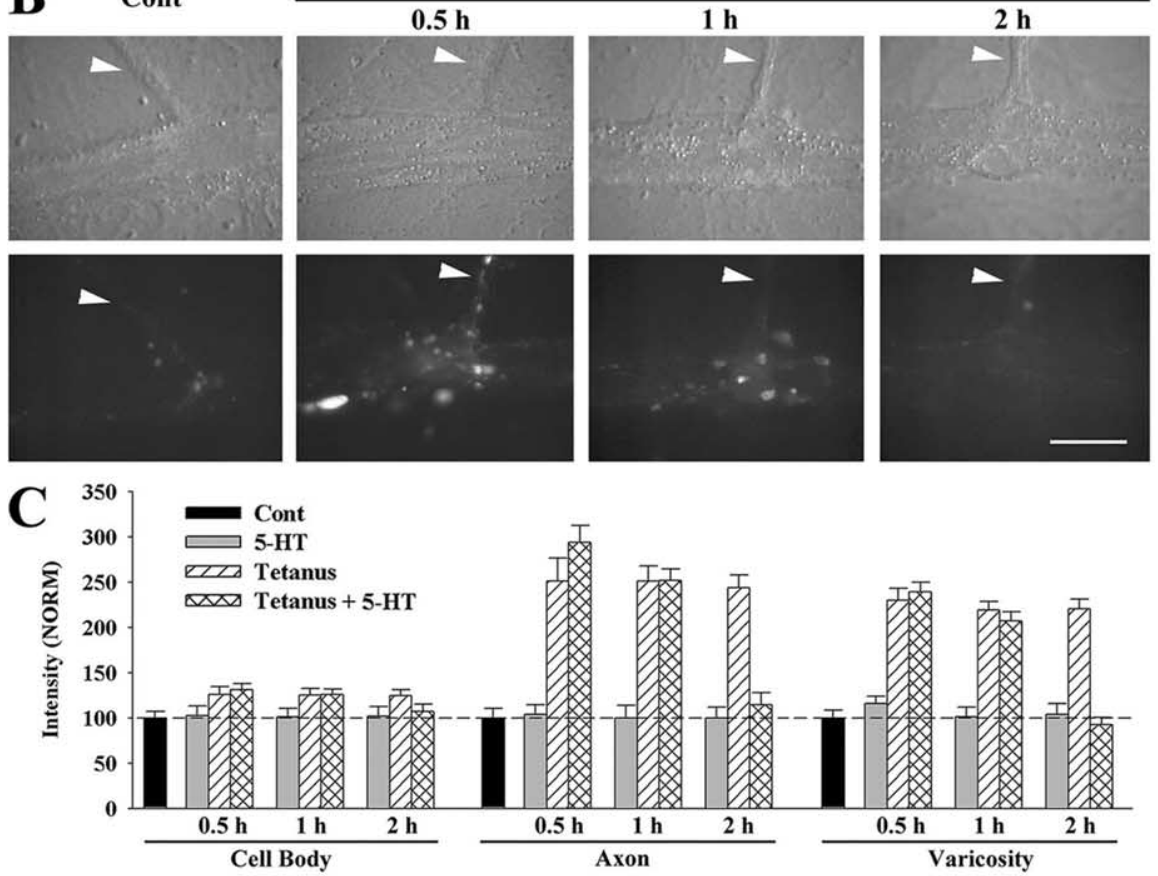

D

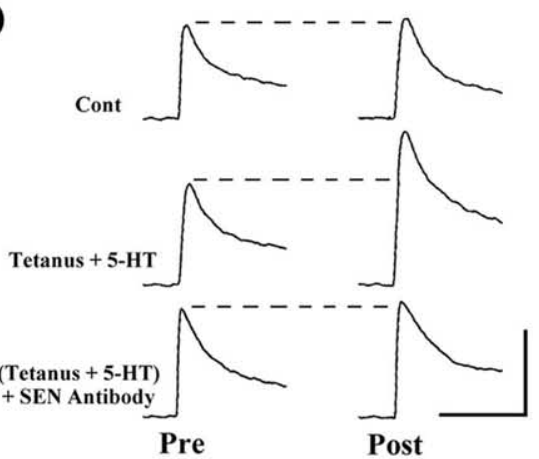

E

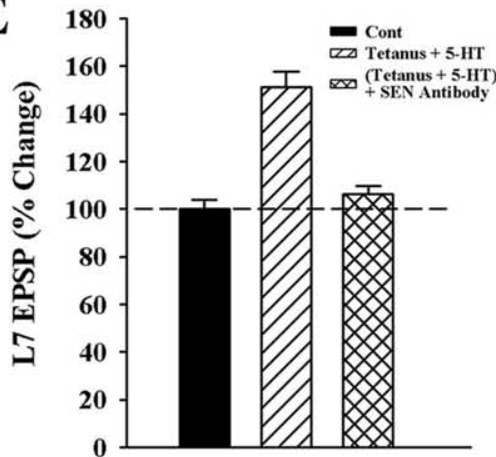

Figure 1. Tetanus plus 5-HT increased both sensorin expression and release required for associative LTF. $\boldsymbol{A}, \boldsymbol{B}$, Nomarski contrast (top) and sensorin immunofluorescent images (bottom) of cocultures $0.5 \mathrm{~h}$ after control (Cont) (mock application) or at $0.5,1$, and $2 \mathrm{~h}$ after tetanus ( $2 \mathrm{~s}$ at $20 \mathrm{~Hz} ; \boldsymbol{A}$ ) or after tetanus plus $5-\mathrm{HT}$ ( 2 sat $20 \mathrm{~Hz}$ plus 5 min application; $\boldsymbol{B}$ ). Staining for sensorin, confined primarily to granules in the sensory neuron axons (arrowheads), regenerated processes or varicosities contacting $\mathrm{L} 7$, increased at $0.5 \mathrm{~h}$ and $1 \mathrm{~h}$ after either stimuli, but decreased to control levels at $2 \mathrm{~h}$ only after tetanus plus $5-\mathrm{HT}$. The axons and processes of L7 are unstained for sensorin. Scale bar, $30 \mu \mathrm{m}$. C, Summary of sensorin immunostaining in cell body, axon, and varicosities of sensory neurons after control (Cont) and at various times after 5 -HT, tetanus, or tetanus plus 5 -HT. Staining in each compartment was normalized to the average staining for that compartment after control (dashed line at 100\%). ANOVA indicated a significant effect of treatment ( $\mathrm{df}=18,162 ; F=48.522 ; p<0.001) .5$ - $\mathrm{HT}$ alone produced no significant change in sensorin staining, but tetanus significantly increased staining in axons and varicosities at $0.5 \mathrm{~h}(F=5.34$ and $F=8.027 ; p<0.01)$, at $1 \mathrm{~h}$ $(F=7.004$ and $F=8.851 ; p<0.01)$ and at $2 \mathrm{~h}(F=6.321$ and $F=9.067 ; p<0.01)$. Tetanus plus 5 - $\mathrm{HT}$ significantly increased staining in axons and varicosities at $0.5 \mathrm{~h}(F=12.976$ and $F=10.72 ; p<0.01)$ and at $1 \mathrm{~h}(F=8.39$ and $F=7.185 ; p<0.01)$. Sensorin staining was no longer significantly different from control or 5 -HT at $2 \mathrm{~h}$ after tetanus plus 5 -HT. Sensorin staining in the sensory neuron cell body increased modestly after tetanus or tetanus plus 5-HT but was not significantly different from control after tetanus plus 5 -HT $(239 \pm 11 \%)$. Staining for sensorin in the cell body increased modestly $(\sim 30 \%)$ but was not significantly different from controls or treatment with 5-HT alone. At $1 \mathrm{~h}$ after tetanus $(n=9)$ or tetanus plus 5-HT $(n=12)$, sensorin staining in the axons and varicosities remained 2- to 2.5-fold higher than control or 5-HT alone in both the axons and distal varicosities. At $2 \mathrm{~h}$ after tetanus alone $(n=9)$, sensorin staining remained nearly 2.5 -fold greater than controls for both axons and varicosities $(244 \pm 14$ and $220 \pm 11 \%)$. In contrast, by $2 \mathrm{~h}$ after tetanus plus 5-HT $(n=10)$ staining for sensorin decreased to control levels both in the axons $(115 \pm 13 \%)$ and varicosities $(92 \pm 8 \%)$. The decline after tetanus plus 5-HT parallel results observed after five applications of 5-HT that produce nonassociative LTF (Hu et al., 2006, their Fig. 1). The results suggest that, although tetanus or tetanus plus 5-HT increased the expression of sensorin, only tetanus plus 5-HT resulted in the rapid disappearance of the newly synthesized sensorin. Because tetanus plus 5-HT produces LTF whereas tetanus alone does not (Schacher et al., 1997; Sun and Schacher, 1998), the secretion of the newly expressed sensorin may account for the reduction in staining and is critical for LTF.

To examine whether the release of the newly expressed sensorin in required for associative LTF, we incubated cultures with anti-sensorin $\mathrm{Ab}$ or control $\mathrm{Ab}$ purified from preimmune serum for $90 \mathrm{~min}$ starting at $0.5 \mathrm{~h}$ after tetanus plus $5-\mathrm{HT}$. Incubation with anti-sensorin Ab blocked associative LTF (Fig. 1D,E). Whereas tetanus plus 5 -HT ( $n=11$ cultures incubated with control $\mathrm{Ab}$ ) produced a significant increase in the EPSP amplitude $(151 \pm 7 \% ; p<0.01) 24 \mathrm{~h}$ after treatment, incubation with anti-sensorin $\mathrm{Ab}(n=13)$

\footnotetext{
$\leftarrow$

or 5 -HT. D, E, Sensorin released after tetanus plus $5-H T$ is required for associative LTF. EPSPs were recorded before (Pre) and $24 \mathrm{~h}$ after (Post) control, tetanus plus 5-HT with control Ab applied between $0.5 \mathrm{~h}$ and $2 \mathrm{~h}$, or tetanus plus 5-HT with anti-sensorin Ab applied between 0.5 and $2 \mathrm{~h}$ (D). The height of the dashed line is the amplitude of each EPSP before treatment. Calibration: $20 \mathrm{mV}, 25 \mathrm{~ms}$. Summary of the changes in EPSPs $24 \mathrm{~h}$ after treatment $(\boldsymbol{E})$. ANOVA indicated a significant effect of treatment ( $\mathrm{df}=2,30 ; F=32.955 ; p<0.001$ ). Tetanus plus 5 -HT with control Ab produced a significant increase in EPSP amplitude compared with control and with tetanus plus 5 - $\mathrm{HT}$ with anti-sensorin $\mathrm{Ab}(F=25.503$ and $F=$ 23.689; $p<0.01$ ). Tetanus plus 5-HT plus anti-sensorin Ab did not significantly affect EPSP amplitude compared with control. The dashed line represents the height of the bar when there was no change in EPSP amplitude.
} 
A
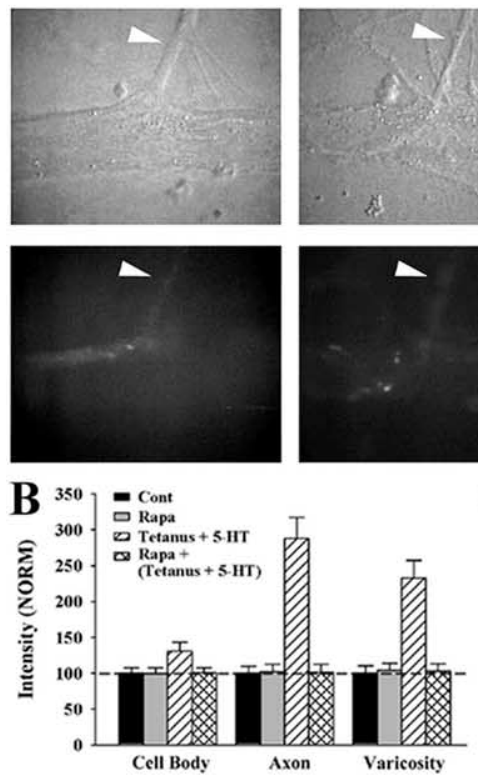

$\mathbf{E}$
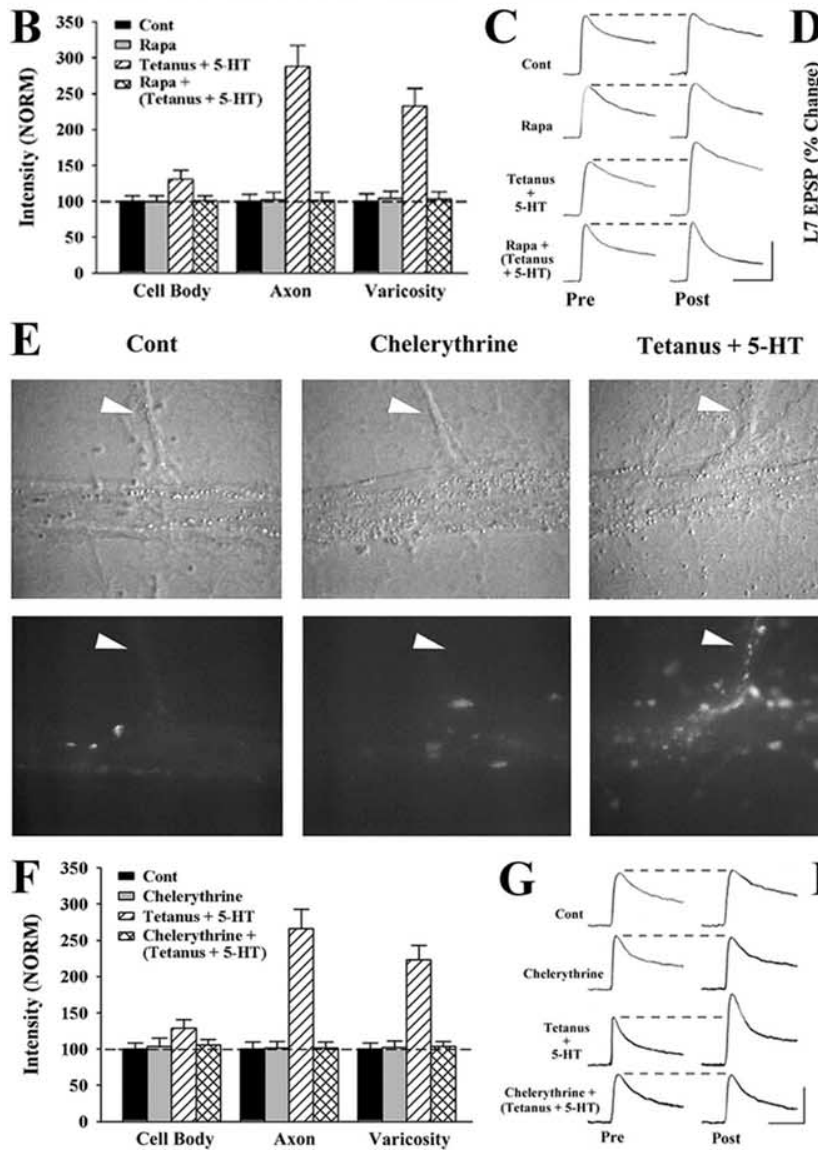

Tetanus + 5-HT
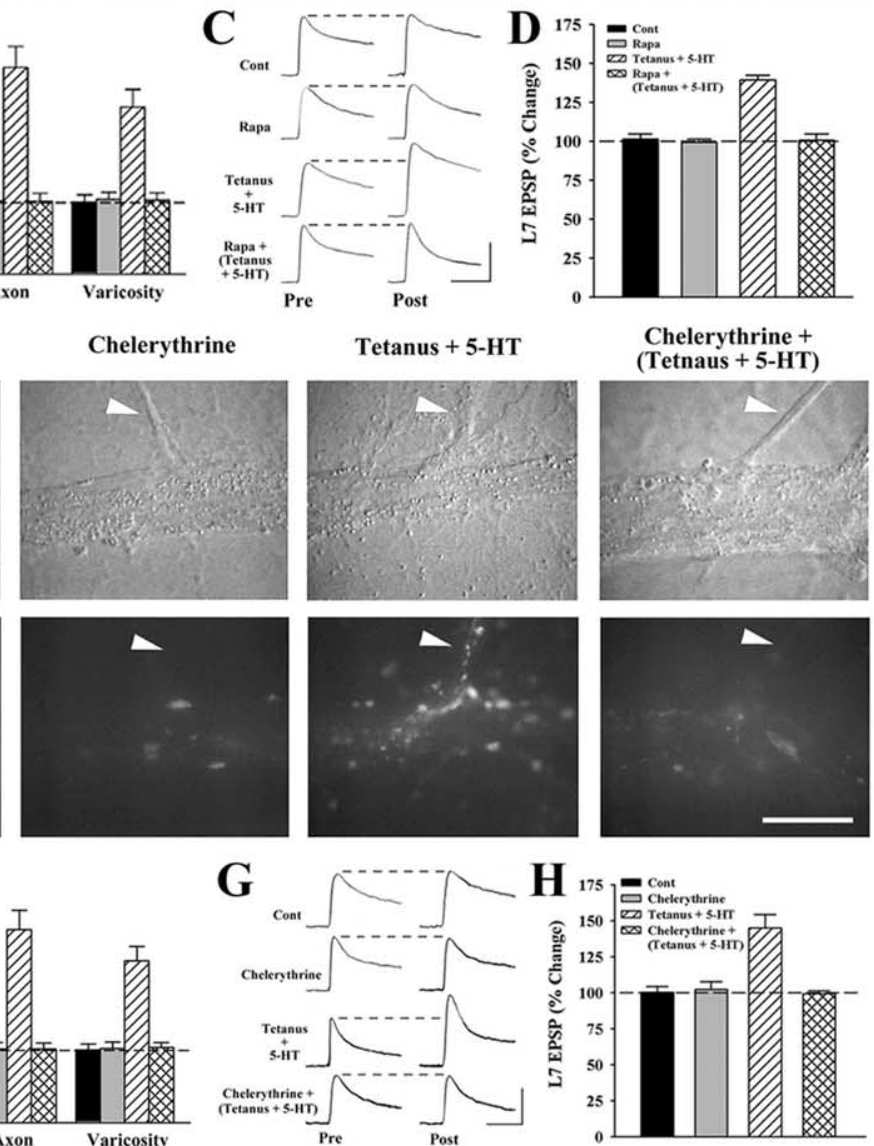

Figure 2. Rapamycin and PKC inhibitor blocked the rapid increase in sensorin and associative LTF produced by tetanus plus 5-HT. A, Nomarski contrast (top) and immunofluorescent images of sensorin staining (bottom) after control (Cont), after 45 min incubation with rapamycin (Rapa), $0.5 \mathrm{~h}$ after tetanus plus 5 - $\mathrm{HT}$, and $0.5 \mathrm{~h}$ after tetanus plus $5-\mathrm{HT}$ with rapamycin. Rapamycin blocked the increase in sensorin staining in both axons (arrowheads) and varicosities produced by tetanus plus 5 -HT. Scale bar, 30 $\mu \mathrm{m}$. $\boldsymbol{B}$, Summary of sensorin immunostaining after treatments. Staining in each compartment was normalized to the average staining after control (dashed line at 100\%). ANOVA indicated a significant effect of treatment (df $=6,44 ; F=10.095 ; p<$ 0.001). Compared with control, tetanus plus $5-\mathrm{HT}$ increased significantly sensorin staining in axons and varicosities $(F=22.131$ and $F=13.874 ; p<0.01)$. In the presence of rapamycin, tetanus plus $5-\mathrm{HT}$ failed to significantly change sensorin staining compared with controls, and change in staining levels were significantly lower than the change in sensorin staining produced by tetanus plus $5-\mathrm{HT}$ in the absence of drug in the axons and varicosities $(F=21.73$ and $F=13.311 ; p<0.01)$. Sensorin staining in the cell body increased modestly after tetanus plus 5 -HT but was not significantly different from control or the other treatments. C, D, Rapamycin blocked associative LTF. EPSPs were recorded before (Pre) and $24 \mathrm{~h}$ after (Post) treatments (C). Calibration: $20 \mathrm{mV}$, $25 \mathrm{~ms}$. Summary of the changes in EPSP amplitudes $24 \mathrm{~h}$ after treatment (D). ANOVA indicated a significant effect of treatment ( $\mathrm{df}=3,20 ; F=35.813 ; p<0.001$ ). Tetanus plus $5-\mathrm{HT}$ evoked a significant increase in EPSP compared with control or tetanus plus 5 -HT with rapamycin $(F=22.682$ and $F=25.448 ; p<0.01)$. Tetanus plus 5 -HT with rapamycin did not significantly affect EPSP amplitudes compared with control or rapamycin alone. $\boldsymbol{E}$, Nomarski contrast (top) and immunofluorescent images of sensorin staining (bottom) immediately after control (Cont), after 45 min incubation with chelerythrine, $0.5 \mathrm{~h}$ after tetanus plus $5-\mathrm{HT}$, and $0.5 \mathrm{~h}$ after tetanus plus $5-\mathrm{HT}$ with chelerythrine. Chelerythrine blocked the increase in sensorin staining in both axons (arrowheads) and varicosities produced by tetanus plus 5 -HT. Scale bar, $30 \mu \mathrm{m}$. $\boldsymbol{F}$, Summary of sensorin immunostaining after treatments. Staining in each compartment was normalized to the average staining after control (dashed line at $100 \%$ ). blocked the increase in EPSP amplitude $(106 \pm 3 \%)$ at $24 \mathrm{~h}$. The change in amplitude was not significantly different from control treatment $(n=9 ; 100 \pm 4 \%)$. Incubation with the antibody for $1 \mathrm{~h}$ after tetanus plus 5-HT did not block associative ITF measured at $1 \mathrm{~h}$ after tetanus plus 5-HT (Sutton and Carew, 2000; Zhao et al., 2006). One hour after tetanus plus 5-HT, EPSP amplitude increased by $191 \pm$ $12 \%(n=10)$. Incubation with antisensorin $\mathrm{Ab}$ during the $1 \mathrm{~h}$ period after stimulation failed to affect the increase in EPSP amplitude; the EPSP amplitude increased by $194 \pm 15 \%(n=14)$. Thus, the secretion of newly expressed sensorin during the period when sensorin level in the axons and varicosities of sensory neurons declines precipitously (between 0.5 to $2 \mathrm{~h}$ after tetanus plus 5-HT) is required for associative LTF but not associative ITF.

\section{Rapid increase in sensorin expression produced by tetanus plus $5-\mathrm{HT}$ is rapamycin sensitive and PKC dependent}

Rapamycin blocks increases in the expression of sensorin after five applications of 5-HT (Hu et al., 2006). Treatment with rapamycin for $45 \mathrm{~min}$ beginning $15 \mathrm{~min}$ before tetanus plus 5-HT also blocked the increase in sensorin staining at $0.5 \mathrm{~h}$ (Fig. $2 A, B)$. In the presence of rapamycin, tetanus plus 5 -HT $(n=7)$ failed to increase sensorin staining compared with controls ( $n=7$; normalized to $100 \%$ for each compartment) in both axons $(102 \pm 11 \%)$ and varicosities $(103 \pm 10 \%)$. Without rapamycin $(n=6)$, tetanus plus 5 -HT increased sensorin staining by nearly threefold in the sensory neuron axons (288 \pm $29 \%)$ and by nearly 2.5 -fold (233 $\pm 25 \%)$ in the varicosities (Fig. $2 A, B$ ). The $45 \mathrm{~min}$

ANOVA indicated a significant effect of treatment $(\mathrm{df}=6,44$; $F=31.174 ; p<0.001)$. Compared with control, tetanus plus 5 -HT produced significant changes in sensorin staining in axons and varicosities $(F=19.454$ and $F=17.267 ; p<0.01)$. In the presence of chelerythrine, tetanus plus 5 -HT failed to change significantly sensorin staining, and staining levels were significantly lower than the changes in sensorin staining produced by tetanus plus 5 -HT without drug both in the axons and varicosities $(F=21.999$ and $F=19.013 ; p<0.01) . \mathbf{G}, \boldsymbol{H}$, Chelerythrine blocked associative LTF. EPSPs were recorded before (Pre) and $24 \mathrm{~h}$ after (Post) treatments (G). Calibration: $20 \mathrm{mV}, 25 \mathrm{~ms}$. Summary of the changes in EPSP amplitudes $24 \mathrm{~h}$ after treatments $(\boldsymbol{H})$. ANOVA indicated a significant effect of treatment $(\mathrm{df}=3,23 ; F=15.387 ; p<0.001)$. Tetanus plus 5 -HT evoked a significant increase in EPSP amplitude compared with control and with tetanus plus 5 -HT with chelerythrine $(F=9.62$ and $F=12.166 ; p<0.01)$. Tetanus plus 5-HT with chelerythrine did not significantly affect EPSP amplitudes compared with control or chelerythrine alone. 

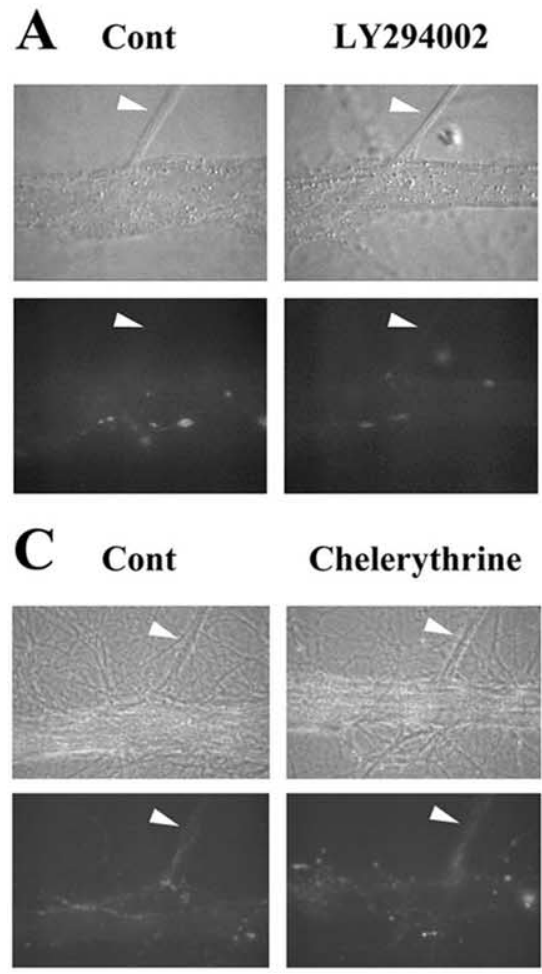
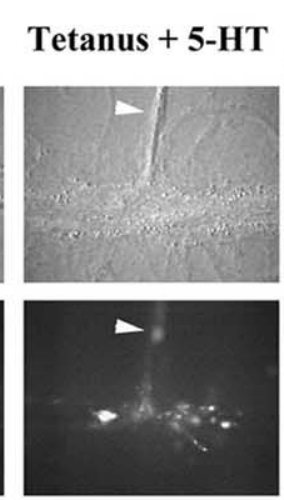

\section{X 5-HT}
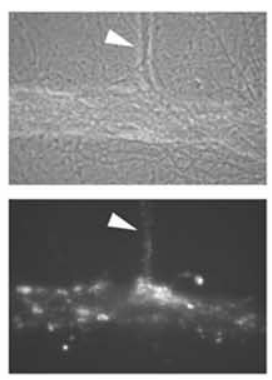

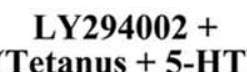

(Tetanus + 5-HT)
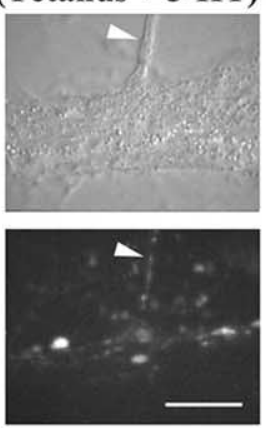

Chelerythrine $+(5 \times 5-H T)$
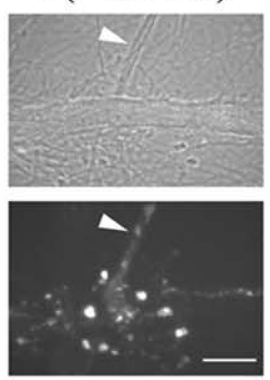
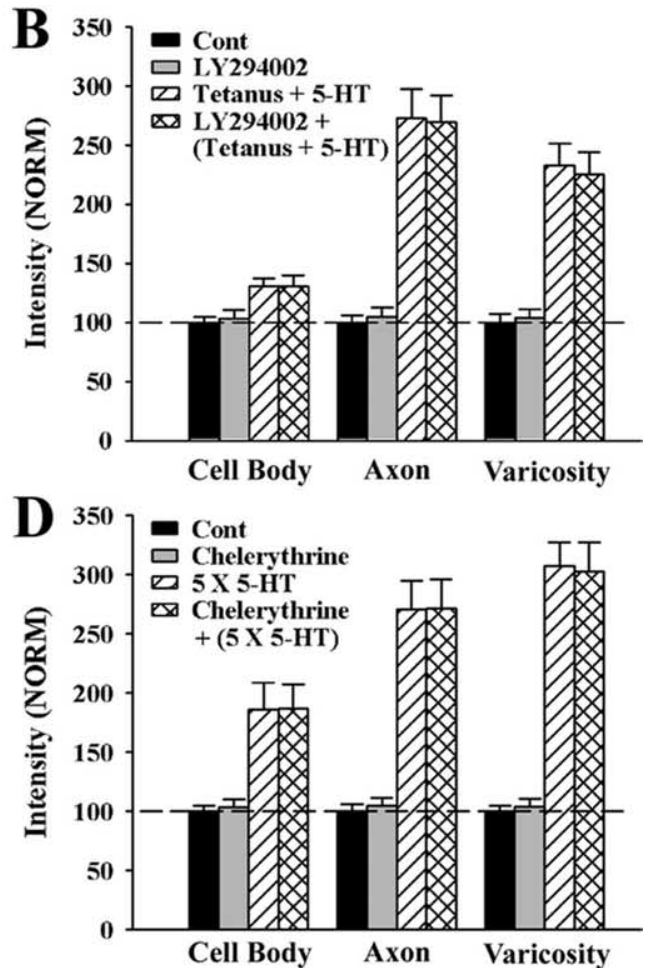

Figure 3. Signaling pathway required for regulating rapid sensorin synthesis differs for associative LTF and nonassociative LTF. $A, B$, PI3K activity is not required for rapid sensorin synthesis after tetanus plus 5-HT. Nomarski contrast (top) and immunofluorescent images of sensorin staining (bottom) after control (Cont), after 45 min incubation with PI3K inhibitor (LY294002), $0.5 \mathrm{~h}$ after tetanus plus 5-HT, and $0.5 \mathrm{~h}$ after tetanus plus 5-HT with LY294002. The inhibitor failed to block the increase in sensorin staining in both axons (arrowheads) and varicosities produced by tetanus plus 5-HT. Scale bar, $30 \mu \mathrm{m}$. B, Summary of sensorin immunostaining after treatments. Staining in each compartment was normalized to the average staining after control (dashed line at 100\%). ANOVA indicated a significant effect of treatment ( $\mathrm{df}=6,44 ; F=28.486 ; p<0.001)$. Compared with control, tetanus plus 5 -HT significantly increased sensorin staining in axons and varicosities $(F=14.78$ and $F=13.332 ; p<0.01)$. In the presence of $L Y 294002$, tetanus plus 5-HT also significantly increased sensorin staining in the axons and varicosities $(F=15.329$ and $F=12.833 ; p<0.01)$. Staining after tetanus plus 5 -HT with drug was not significantly different from the staining detected after tetanus plus 5 -HT without drug. Sensorin staining in the cell body increased modestly after tetanus plus 5-HT but was not significantly different from control or the other treatments. C, D, PKC activity is not required for the increase in sensorin after five applications of 5 -HT. Nomarski contrast (top) and immunofluorescent images of sensorin staining (bottom) after control (Cont), after $2 \mathrm{~h}$ incubation with chelerythrine, immediately after five applications of 5-HT, and immediately after five applications of 5-HT plus chelerythrine. The inhibitor failed to block the increase in sensorin staining in both axons (arrowheads) and varicosities produced by five applications of 5-HT. Scale bar, $30 \mu \mathrm{m} . B$, Summary of sensorin immunostaining after treatments. Staining in each compartment was normalized to the average staining after control (dashed line at $100 \%$ ). ANOVA indicated a significant effect of treatment ( $\mathrm{df}=6,44 ; F=41.679 ; p<0.001)$. Compared with control, five applications of 5 -HT significantly increased sensorin staining in cell bodies $(F=4.272 ; p<0.05)$ and axons and varicosities $(F=13.421$ and $F=23.232 ; p<0.01)$. In the presence of chelerythrine, tetanus plus 5 - $H T$ also significantly increased sensorin staining in the cell bodies $(F=4.343 ; p<$ 0.05 ) and axons and varicosities $(F=13.602$ and $F=22.281 ; p<0.01)$. Staining after five applications of 5 -HT with drug was not significantly different from the staining after five applications of 5 -HT without drug.

treatment with rapamycin alone $(n=6)$ did not significantly affect sensorin staining compared with controls $(102 \pm 11 \%$ in the axons and $104 \pm 10 \%$ in the varicosities). Rapamycin also blocked the increase in sensorin staining in axons and varicosities produced by tetanus alone (data not shown). Thus, the rapid increase in sensorin in both axons and varicosities depend on rapamycin-sensitive protein synthesis.

The same treatment with rapamycin also blocked associative LTF produced by tetanus plus 5-HT (Fig. 2C,D). Whereas tetanus plus 5 -HT $(n=6)$ produced a significant increase in the EPSP amplitude $(140 \pm 3 \%) 24 \mathrm{~h}$ after stimulation compared with controls $(n=6 ; 102 \pm 3 \%)$, rapamycin $(n=7)$ blocked the increase in EPSP amplitude $(101 \pm 4 \%)$ at $24 \mathrm{~h}$ after tetanus plus 5 -HT. The change in amplitude was not significantly different from the change with rapamycin treatment alone $(n=5 ; 99 \pm$ $2 \%$ ). Thus, associative LTF that is accompanied by structural plasticity (Sun and Schacher, 1998) and is produced by a cellwide application of 5-HT requires rapamycin-sensitive protein synthesis that includes the synthesis of sensorin (for protein synthesis-independent form of associative LTF produced by tetanus plus application of 5-HT only at the terminal region of the sensory neuron and the motor neuron, see Bailey et al., 2000).
Protein kinase C inhibitor chelerythrine, which fails to block nonassociative LTF when applied during the five applications of 5-HT (Hu et al., 2003, 2004a), blocked the rapid increase in sensorin synthesis produced by tetanus plus 5-HT (Fig. 2E,F). Treatment with chelerythrine for $45 \mathrm{~min}$ beginning $15 \mathrm{~min}$ before tetanus plus 5-HT $(n=8)$ reduced significantly the increase in sensorin staining in both the axons $(101 \pm 8 \%)$ and the varicosities $(103 \pm 7 \%)$. In contrast, tetanus plus 5 -HT without the inhibitor $(n=6)$ produced significant increases in sensorin staining in both the axons $(266 \pm 27 \%)$ and varicosities $(224 \pm 20 \%)$. Compared with controls ( $n=6$; normalized to $100 \%$ ), chelerythrine alone $(n=6)$ did not affect sensorin expression in axons $(101 \pm 9 \%)$ or varicosities $(103 \pm 9 \%)$. Chelerythrine also blocked the increases in sensorin staining in the axons and varicosities produced by tetanus alone (data not shown). Thus, the synthesis of sensorin is regulated by two signaling pathways: PI3K activity with five applications of 5-HT and PKC activity with tetanus plus 5-HT (see below).

The brief incubation with chelerythrine also blocked associative LTF produced by tetanus plus 5-HT (Fig. 2G,H). Whereas tetanus plus 5 -HT $(n=7)$ produced a significant increase in the EPSP amplitude (145 $\pm 9 \%) 24 \mathrm{~h}$ after stimulation compared 
with controls $(n=6 ; 100 \pm 4 \%)$, chelerythrine $(n=9)$ blocked the increase in EPSP amplitude $(99 \pm 2 \%)$ at $24 \mathrm{~h}$ after tetanus plus 5 -HT. The change in amplitude was not significantly different from the change with chelerythrine alone $(n=5 ; 102 \pm 5 \%)$. Thus, associative LTF produced by tetanus plus 5 -HT requires PKC activity $15 \mathrm{~min}$ before, during, and $30 \mathrm{~min}$ after stimuli. In contrast, PKC activity immediately before, during, and immediately after five applications of 5-HT is not required for nonassociative LTF.

The rapid increase in sensorin synthesis with five applications of 5-HT requires PI3K activity (Hu et al., 2006). Does PI3K activity contribute to the increase in sensorin expression after tetanus plus 5-HT? PI3K inhibitor had no effect on the rapid increase in sensorin staining after tetanus plus 5-HT (Fig. $3 A, B$ ). We incubated cultures with the PI3K inhibitor LY294002 for $45 \mathrm{~min}$ beginning $15 \mathrm{~min}$ before pairing tetanus plus 5-HT. In the presence of LY294002 $(n=7)$, tetanus plus 5-HT increased sensorin staining by $>2.5$-fold in axons $(269 \pm 23 \%)$ and nearly 2.5 -fold in varicosities $(225 \pm 19 \%)$ compared with controls $(n=6$; normalized to $100 \%)$. These increases were comparable with the increase in sensorin staining in axons $(273 \pm 25 \%)$ and varicosities $(233 \pm 18 \%)$ after tetanus plus 5-HT without the inhibitor $(n=6)$. Incubation with the PI3K inhibitor alone $(n=7)$ did not significantly affect sensorin staining in axons $(104 \pm 8 \%)$ or in varicosities $(103 \pm 7 \%)$. As is the case with five applications of 5-HT (Hu et al., 2006), incubation with the PKA inhibitor KT5720 for 45 min beginning 15 min before tetanus plus 5-HT also failed to block the rapid increase in sensorin expression (data not shown). Thus, PI3K activity, which plays a crucial role in regulating the rapid synthesis of sensorin with nonassociative LTF produced by five applications of 5-HT (Hu et al., 2006), is not essential for regulating the rapid increase in sensorin after tetanus plus 5 -HT.

Although blocking PKC activity during five applications of 5-HT applications fails to block nonassociative LTF, does it reduce the rapid increase in sensorin synthesis that is observed immediately after five applications of 5-HT? Treatment with chelerythrine beginning $15 \mathrm{~min}$ before and during five applications of 5-HT (total treatment of $\sim 2 \mathrm{~h}$ ) failed to block the increase in sensorin staining detected immediately after treatment (Fig. $3 C, D)$. In the presence of the PKC inhibitor $(n=7)$, five applications of 5-HT increased sensorin staining significantly over control ( $n=6$; normalized to $100 \%)$ in each compartment: nearly twofold in the sensory neuron cell body $(188 \pm 21 \%)$ and nearly threefold both in the axons $(272 \pm 24 \%)$ and in the varicosities $(303 \pm 24 \%)$. These changes were comparable with the increases in sensorin staining after five applications of 5-HT without the PKC inhibitor $(n=7 ; 186 \pm 23 \%$ in the cell body, $270 \pm 24 \%$ in the axons, and $308 \pm 20 \%$ in the varicosities). Incubation with the inhibitor alone for $2 \mathrm{~h}(n=6)$ did not affect sensorin staining in all compartments compared with controls (103 \pm 7 in the cell body, $105 \pm 6$ in the axons, and $104 \pm 7$ in the varicosities). Thus, blocking PKC activity, which blocks the rapid increase in sensorin with associative LTF (tetanus plus 5-HT), does not interfere with the rapid increase in sensorin with nonassociative LTF (five applications of 5-HT).

Because blocking PKC activity blocks the increase in sensorin after tetanus or tetanus plus 5-HT, we next examined whether activating $\mathrm{PKC}$ produces an increase in sensorin. Brief incubation ( $5 \mathrm{~min}$ ) with PDBu produced a significant increase in sensorin at $0.5 \mathrm{~h}$ (Fig. 4). Compared with control untreated cultures $(n=6$; normalized to $100 \%$ for each compartment), brief exposure to $\mathrm{PDBu}(n=8)$ increased sensorin staining in the sensory neuron
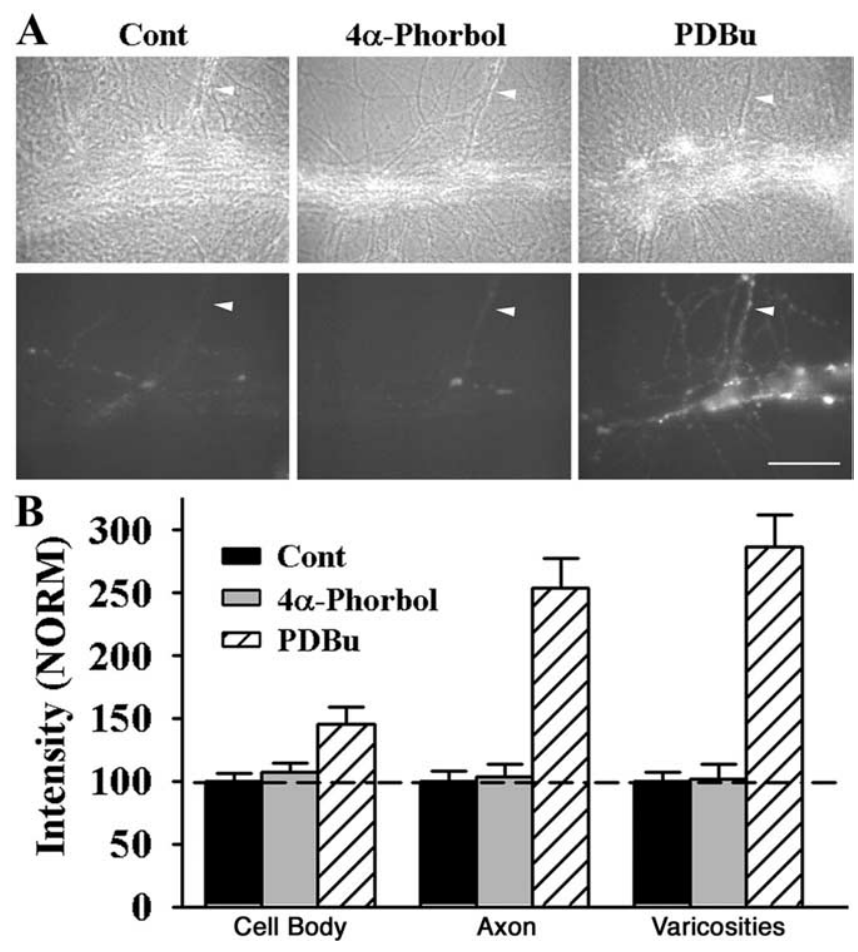

Figure 4. PDBu rapidly increased sensorin in sensory neurons. $\boldsymbol{A}$, Phase contrast (top) and immunofluorescent images of sensorin staining (bottom) in sensory neuron axons (arrowheads) and varicosities immediately after control treatment (Cont), $0.5 \mathrm{~h}$ after $5 \mathrm{~min}$ application of inactive phorbol ( $4 \alpha$-phorbol), and $0.5 \mathrm{~h}$ after $5 \mathrm{~min}$ application of PDBu. Sensorin staining increased in the axon and varicosities only after treatment with PDBu. Scale bar, $50 \mu \mathrm{m}$. $\boldsymbol{B}$, Summary of sensorin immunostaining after treatments. Staining in each compartment was normalized to the average staining after control (dashed line at 100\%). ANOVA indicated a significant effect of treatment ( $\mathrm{df}=4,34 ; F=51.524 ; p<0.001$ ). PDBu significantly increased sensorin staining compared with control or $4 \alpha$-phorbol in the sensory neuron cell bodies $(F=5.76, p<0.03$; or $F=4.195, p<0.05)$, axons $(F=23.379$ or $F=22.376$; $p<$ $0.01)$, and varicosities $(F=26.766$ or $F=25.337 ; p<0.01)$. Treatment with $4 \alpha$-phorbol did not significantly affect sensorin staining compared with control.

cell body by nearly $50 \%$ ( $146 \pm 12 \%)$, by $>2.5$-fold in the axons $(254 \pm 21 \%)$, and by nearly threefold in the varicosities $(286 \pm$ $24 \%)$. Brief exposure to the inactive $4 \alpha$-phorbol $(n=6)$ failed to significantly affect sensorin staining $(107 \pm 7 \%$ in the cell body, $103 \pm 10 \%$ in the axon, and $102 \pm 10 \%$ in the varicosities). Thus, activating PKC produces a rapid increase in sensorin synthesis throughout the sensory neuron.

\section{Sensory neuron cell body is not required for the rapid} synthesis of sensorin in axon and varicosities

The rapid and significant increase in sensorin at distal varicosities by $0.5 \mathrm{~h}$ after stimulation suggested that local translation of sensorin mRNA, which accumulates at varicosities after being transported down sensory neuron axons (Schacher et al., 1999; Hu et al., 2002, 2006; Lyles et al., 2006), contributes to local changes in sensorin levels. Local synthesis of sensorin is detected after removing the sensory neuron cell body; five applications of 5-HT produced a rapid rapamycin-sensitive and PI3K-dependent increase in sensorin synthesis in both the axons and varicosities (Hu et al., 2006). We therefore tested whether, after removing the sensory neuron cell bodies, tetanus plus 5 -HT produces a rapid rapamycin-sensitive and $\mathrm{PKC}$-dependent change in the expression of sensorin in the axons and distal varicosities.

Tetanus plus 5-HT produced a significant increase in sensorin in both the axons and the distal varicosities after removal of the 
A
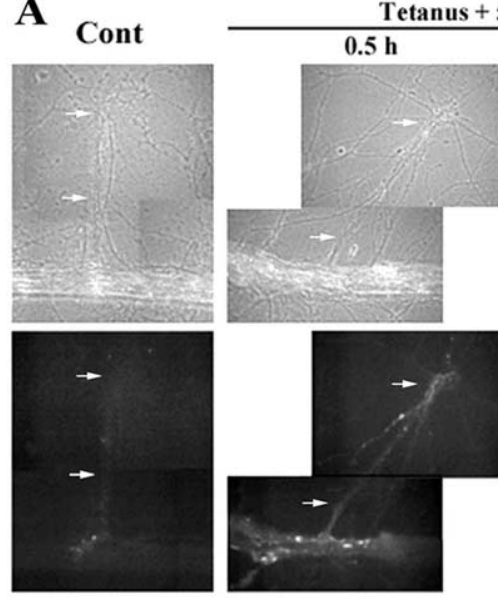

$\mathbf{C}_{\text {Cont }}$

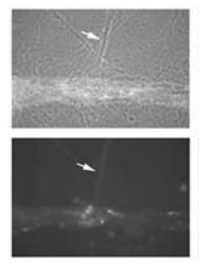

Tetanus + 5-HT
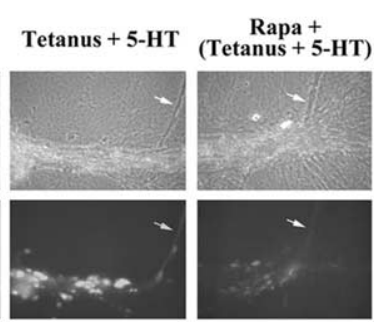
Chelerythrine +
(Tetanus + 5-HT)

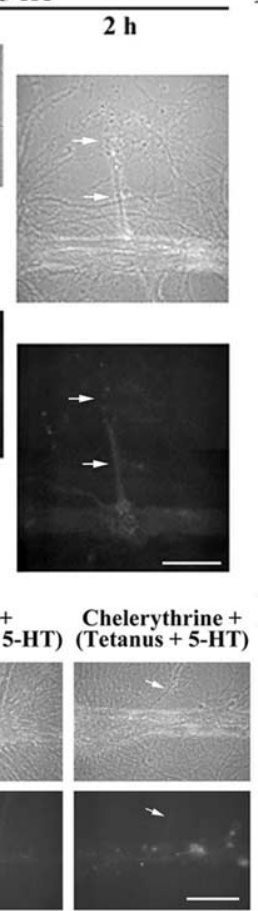

B
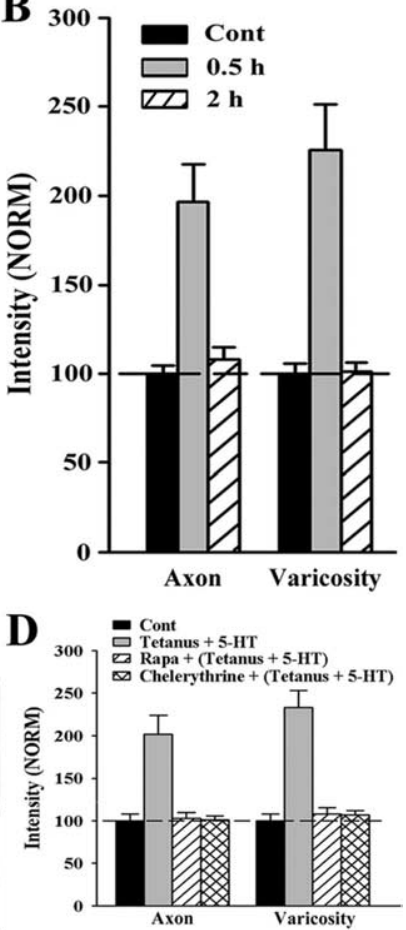

Figure 5. Rapamycin and PKC inhibitor blocked the increase in sensorin produced by tetanus plus $5-\mathrm{HT}$ in the absence of the sensory neuron cell body. $A, B$, Without sensory neuron cell bodies, tetanus plus 5 -HT rapidly increased sensorin at $0.5 \mathrm{~h}$ that recovered to control levels by $2 \mathrm{~h}$. Phase contrast $(\boldsymbol{A}$, top) and immunofluorescent images of sensorin staining $(\boldsymbol{A}$, bottom) in sensory neuron axons (bottom arrows in each image) and varicosities after removal of each sensory neuron cell body (former position indicated by the top arrow in each image). After recovery, cultures were treated either with tetanus plus 5 -HT or control (Cont). Sensorin staining increased at $0.5 \mathrm{~h}$ after tetanus plus 5 -HT and returned to control levels by $2 \mathrm{~h}$ after stimuli. Scale bar, 50 $\mu \mathrm{m}$. Summary of sensorin staining in axons and varicosities of sensory neurons without cell bodies after control and 0.5 and $2 \mathrm{~h}$ after tetanus plus 5 -HT $(\boldsymbol{B})$. Staining in each compartment was normalized to the average staining after control (dashed line at $100 \%$ ). ANOVA indicated a significant effect of treatment ( $\mathrm{df}=2,17 ; F=6.83 ; p<0.007)$. Tetanus plus 5 -HT significantly increased sensorin staining at $0.5 \mathrm{~h}$ compared with control in both the axons and varicosities $(F=11.86$ and $F=14.599 ; p<$ 0.01). Sensorin staining in both axons and varicosities $2 \mathrm{~h}$ after tetanus plus $5-\mathrm{HT}$ was not significantly different from control and was significantly lower than the staining at $0.5 \mathrm{~h}$ after tetanus plus 5 - $\mathrm{HT}$ in both the axons and varicosities $(F=10.767$ and $F=$ $15.53 ; p<0.01)$. C, $\boldsymbol{D}$, Rapamycin (Rapa) and PKC inhibitor blocked the increase in sensorin staining at $0.5 \mathrm{~h}$ after tetanus plus 5 -HT. Phase contrast ( $\boldsymbol{C}$, top) and immunofluorescent images of sensorin staining ( $\boldsymbol{C}$, bottom) of cocultures without sensory neuron cell bodies after control application (cont), $0.5 \mathrm{~h}$ after tetanus plus $5-\mathrm{HT}, 0.5 \mathrm{~h}$ after tetanus plus 5 - $\mathrm{HT}$ with rapamycin, or $0.5 \mathrm{~h}$ after tetanus plus 5 - $\mathrm{HT}$ with chelerythrine. Sensorin staining in axons and varicosities increased only after tetanus plus 5 -HT without drug. Scale bar, $50 \mu \mathrm{m}$. Summary of sensorin staining in axons and varicosities of sensory neurons without cell bodies after control and $0.5 \mathrm{~h}$ after tetanus plus 5 -HT with or without drug $(\boldsymbol{D})$. Staining in each compartment was normalized to the average staining after control (dashed line at 100\%). ANOVA indicated a significant effect of treatment ( $\mathrm{df}=3,22 ; F=5.794$; $p<0.005$ ). Tetanus plus 5 - $\mathrm{HT}$ significantly increased sensorin staining at $0.5 \mathrm{~h}$ compared with control in both the axons and varicosities $(F=9.803$ and $F=19.429 ; p<0.01$ ). Tetanus plus 5 -HT with either drug did not significantly affect sensorin staining in the axons or varicosities. Tetanus plus 5-HT without drug increase sensorin staining compared with tetanus plus 5-HT with rapamycin in both axons and varicosities $(F=9.296$ and $F=17.253 ; p<0.01)$ and compared with tetanus plus 5 -HT with chelerythrine in both axons and varicosities $(F=10.324$ and $F=19.019 ; p<0.01)$.

sensory neuron cell bodies (Fig. 5A,B). Compared with control $(n=6 ; 100 \%)$, tetanus (applied to axon stump) plus 5-HT ( $n=$ 7) significantly increased sensorin staining at $0.5 \mathrm{~h}$ by nearly twofold in axons $(196 \pm 21 \%)$ and by nearly 2.5 -fold in varicosities $(226 \pm 26 \%)$. As was observed for intact sensory neurons (Fig. 1), sensorin staining returned to control levels by $2 \mathrm{~h}$ after tetanus plus 5 -HT $(n=7)$ in both the axons $(108 \pm 6 \%)$ and varicosities $(101 \pm 5 \%)$. This indicates that local translation of sensorin and the local release of the newly synthesized sensorin may contribute to associative LTF.

Although tetanus plus 5-HT increases sensorin in both the axons and varicosities even in the absence of sensory neuron cell bodies, these changes require the presence of the motor neuron L7. Stimulating sensory neurons plated without L7 with tetanus plus 5 -HT failed to significantly alter sensorin expression at $0.5,1$, or $2 \mathrm{~h}(n=4$ for each time point) after stimulation (data not shown). However, incubating sensory neurons for $5 \mathrm{~min}$ with $\mathrm{PDBu}(n=10)$ compared with incubation with $4 \alpha$ phorbol $(n=8$; normalized to $100 \%)$ significantly increased sensorin staining at 0.5 h by $144 \pm 14 \%$ in the cell body, $157 \pm$ $11 \%$ in the axons, and $150 \pm 13 \%$ in the distal neurites and varicosities. Although significant, the increases in sensorin expression in the axon and distal neurites and varicosities were lower than the increases produced by PDBu when sensory neurons contact L7 (Fig. 4). Thus, although tetanus plus 5-HT failed to increase sensorin expression, bypassing the stimulus-induced activation of PKC by direct activation of $\mathrm{PKC}$ with phorbol regulated the rapid expression of sensorin in isolated sensory neurons but at a lower level than observed when sensory neurons formed synapses with L7.

Because rapamycin and chelerythrine block the increases in sensorin in the intact sensory neuron after tetanus plus 5-HT, we examined whether the inhibitors interfered with the increases in sensorin staining at $0.5 \mathrm{~h}$ when tetanus plus $5-\mathrm{HT}$ was applied after removing the sensory neuron cell bodies. The inhibitors, applied for 45 min beginning $15 \mathrm{~min}$ before stimulation, interfered with the local increases in sensorin staining produced by tetanus plus 5-HT (Fig. 5C,D). Compared with control $(n=6 ; 100 \%)$, rapamycin blocked the increase in sensorin staining produced by tetanus plus 5-HT $(n=6)$ in both the axons $(103 \pm 7 \%)$ and varicosities $(107 \pm$ $5 \%)$. The PKC inhibitor also blocked the increases in sensorin staining produced by tetanus plus 5-HT $(n=7)$ in both the axons $(101 \pm 4 \%)$ and varicosities $(107 \pm$ $6 \%$ ). Without drugs, tetanus plus 5-HT $(n=7)$ produced a significant increase in sensorin staining in both the axons (202 \pm $22 \%)$ and varicosities $(233 \pm 20 \%)$. Thus, much of the increase in sensorin in axons and varicosities is produced by the actions of paired stimuli on the rapamycin-sensitive and PKC-dependent pathways that regulate local translation of sensorin mRNA present in axons and varicosities of sensory neurons. In addition, the disappearance of the newly synthesized sensorin by $2 \mathrm{~h}$ suggests that the machinery for packaging and releasing the newly synthesized neuropeptide is present at the distal sites.

\section{Both PKA and PKC activities regulate the secretion of newly synthesized sensorin}

The rapid release of the newly synthesized sensorin after five applications of 5-HT or tetanus plus 5-HT is required for nonassociative or associative LTF (Hu et al., 2004a, 2006) (Fig. 1). Type II PKA activity is required for the release of the newly synthesized 
sensorin after five applications of 5-HT (Hu et al., 2006). We therefore examined whether blocking the signaling pathways affected the release of sensorin after tetanus plus 5-HT. Blocking either PKA activity or PKC activity blocked both the apparent release of sensorin and associative LTF.

Blocking activity of all PKA isoforms with KT5720 blocked the decline of the newly synthesized sensorin in axons and varicosities (Fig. 6A,B). Applying KT5720 between 0.5 and $2 \mathrm{~h}$ after stimulation interfered with the reduction in sensorin staining that normally occurs after tetanus plus 5 -HT (Fig. $6 A, B$ ). The nearly 2.5 -fold increase in sensorin staining in both the axons (236 $\pm 21 \%)$ and varicosities $(265 \pm 24 \%)$ at $0.5 \mathrm{~h}$ after tetanus plus 5 -HT $(n=6)$ failed to decline by $2 \mathrm{~h}$ after stimulation in the presence of the drug $(229 \pm 15 \%$ in axons and $251 \pm 24 \%$ in varicosities). In the absence of KT5720 $(n=6)$, sensorin staining in the axons declined to $105 \pm 13 \%$ and in the varicosities declined to $100 \pm 11 \%$ by $2 \mathrm{~h}$ after tetanus plus 5-HT. A similar result was obtained when cultures were incubated for $90 \mathrm{~min}$ starting $0.5 \mathrm{~h}$ after stimuli with the membrane-permeable form of a peptide sHt-31 that interferes with the interactions between type II PKA and AKAPs. Sensorin staining that increased by $>2.5$ fold in both the axons and varicosities $0.5 \mathrm{~h}$ after tetanus plus 5-HT failed to decline when cultures were incubated with the active peptide sHt-31 $(n=7 ; 231 \pm$ $17 \%$ in axons and $243 \pm 22 \%$ in varicosities) but returned to control levels $(n=6$; $100 \%$ ) when cultures were incubated with the permeable control peptide sHt-31P that has a single amino acid substitution $(n=6 ; 108 \pm 11 \%$ in axons and $112 \pm$ $15 \%$ in varicosities). Associative LTF was blocked when cultures were incubated with KT5720 between 0.5 and $2 \mathrm{~h}$ (Fig. $6 C, D)$. In the presence of the drug $(n=8)$, tetanus plus 5-HT failed to increase significantly EPSP amplitudes $24 \mathrm{~h}$ later (109 \pm $3 \%$ compared with $165 \pm 7 \%$ produced by tetanus plus 5-HT without KT5720; $n=$ 7). Associative LTF was also blocked by incubation with the permeable peptide sHt-31 that interferes with type II PKAAKAP interaction. At $24 \mathrm{~h}$ after stimulation, EPSP amplitudes changed by only $105 \pm 5 \%(n=6)$. Associative LTF was produced by tetanus plus 5 -HT when cultures were incubated with the control peptide sHt-31P $(n=6 ; 149 \pm 6 \%)$. Thus, type II PKA activity is critical for the release of the newly synthesized sensorin and is required for associative LTF.
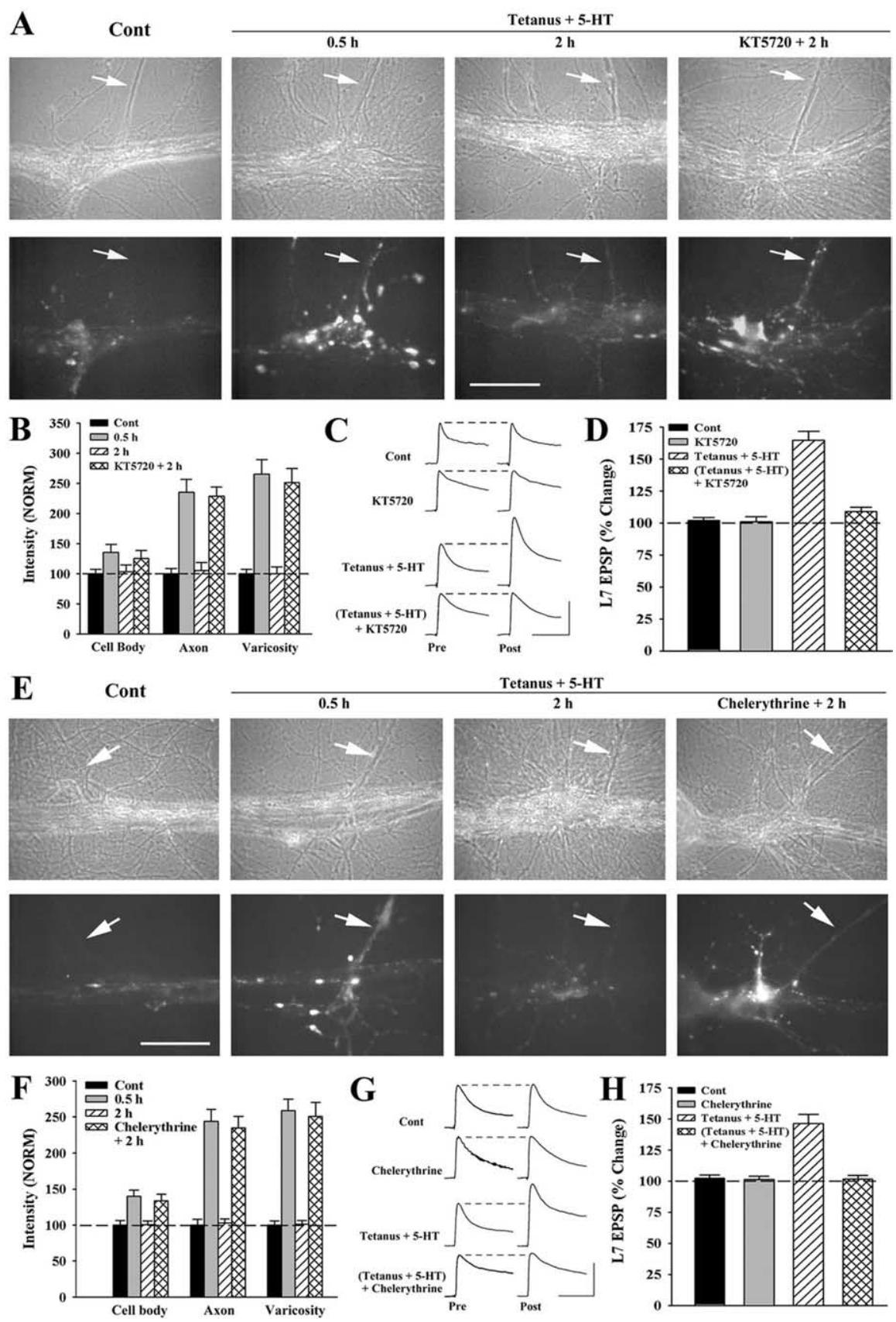

Figure 6. Both PKA and PKC activities are required for the release of sensorin after tetanus plus 5-HT and associative LTF. $A, B$, PKA inhibitor blocked the decline in sensorin staining $2 \mathrm{~h}$ after tetanus plus 5 - $\mathrm{HT}$. Phase contrast $(\boldsymbol{A}, \mathrm{top})$ and immunofluorescent images of sensorin staining ( $\boldsymbol{A}$, bottom) after control (Cont), $0.5 \mathrm{~h}$ after tetanus plus 5-HT, $2 \mathrm{~h}$ after tetanus plus 5 - $\mathrm{HT}$, and $2 \mathrm{~h}$ after tetanus plus 5-HT with KT5720 present between 0.5 and $2 \mathrm{~h}$. The rapid increase in sensorin staining in axons (arrows) and varicosities at $0.5 \mathrm{~h}$ does not decline at $2 \mathrm{~h}$ with the PKA inhibitor. Scale bar, $50 \mu \mathrm{m}$. Summary of the changes in sensorin staining after treatments $(\boldsymbol{B})$. Staining in each compartment was normalized to the average staining after control (dashed line at $100 \%$ ). ANOVA indicated a significant effect of treatment $(\mathrm{df}=6,40 ; F=37.858 ; p<0.001)$. Compared with control, tetanus plus 5-HT at $0.5 \mathrm{~h}$ produced a significant increase in sensorin staining in both the axons and varicosities $(F=13.208$ and $F=13.86 ; p<$ 0.01). Without PKA inhibitor, sensorin staining in all compartments at $2 \mathrm{~h}$ after tetanus plus 5 - $\mathrm{HT}$ was not significantly different from control. Sensorin staining at $2 \mathrm{~h}$ after tetanus plus 5 -HT with KT5720 was not significantly different from the staining at $0.5 \mathrm{~h}$ after tetanus plus 5-HT, remained significantly higher than control in both the axons and varicosities $(F=11.941$ and $F=11.61$; $p<0.01$ ), and was significantly higher than the staining $2 \mathrm{~h}$ after tetanus plus 5 -HT without drug both in the axons and varicosities $(F=11.002$ and $F=11.16 ; p<0.01)$. C, D, PKA inhibition between 0.5 and $2 \mathrm{~h}$ after tetanus plus 5 -HT blocked associative LTF. EPSPs were recorded before (Pre) and $24 \mathrm{~h}$ after (post) control, $90 \mathrm{~min}$ of KT5720, tetanus plus 5-HT, and tetanus plus 5 -HT with KT5720 added between 0.5 and $2 \mathrm{~h}$ after paired stimuli (C). Calibration: $20 \mathrm{mV}, 25 \mathrm{~ms}$. Summary of the changes in EPSP amplitudes $24 \mathrm{~h}$ after treatment $(\boldsymbol{D})$. ANOVA indicated a significant effect of treatment $(\mathrm{df}=3,23 ; F=145.627 ; p<0.001)$. Change after tetanus plus 5-HT with KT5720 was not significantly different from control or treatment with KT5720 alone but was significantly lower than the increase produced tetanus plus 5-HT without drug ( $F=26.509 ; p<0.01)$. Tetanus plus 5-HT significantly increased EPSP amplitude compared with control $(F=29.108 ; p<0.01)$. E, $\boldsymbol{F}$, PKC (Figure legend continues) 

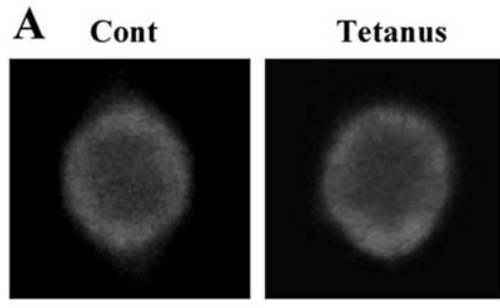

Tetanus $+5-\mathrm{HT}$

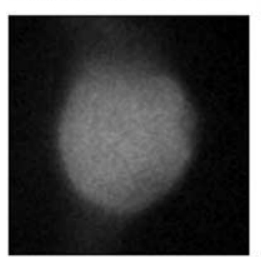

Tetanus + 5-HT $+($ SEN Antibody)

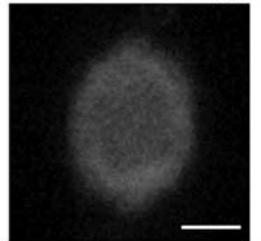

B

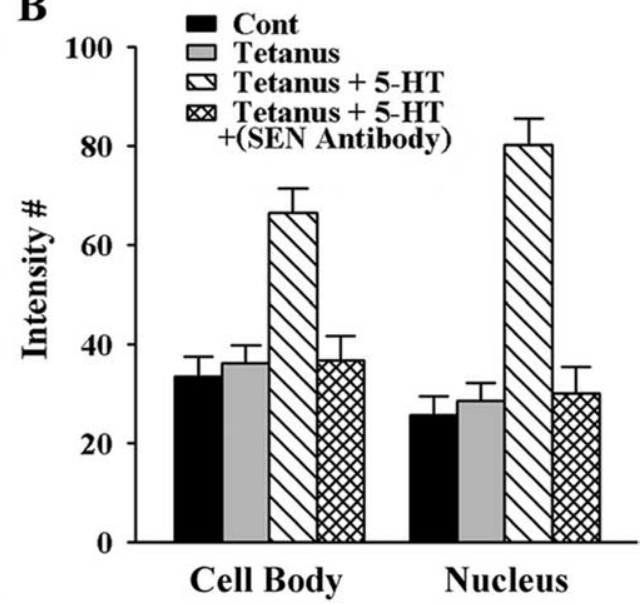

Figure 7. Activation and translocation of p42/44 MAPK in the cell bodies of sensory neurons $1 \mathrm{~h}$ after tetanus plus 5 -HT is blocked by anti-sensorin Ab. A, Immun ofluorescent images of phospho MAPK staining in the cell body of sensory neurons $1 \mathrm{~h}$ after control (Cont), tetanus, tetanus plus 5-HT, and tetanus plus 5-HT with incubation for $1 \mathrm{~h}$ with anti-sensorin (SEN) Ab. Note the increase in overall staining in the cell body and staining in the nucleus only after tetanus plus 5 -HT. Scale bar, $25 \mu \mathrm{m}$. $\boldsymbol{B}$, Summary of phospho-MAPK immunostaining in the whole cell body and nucleus after treatments. ANOVA indicated a significant effect of treatment ( $\mathrm{df}=3,38 ; F=35.255 ; p<0.001$ ). Tetanus plus 5 -HT significantly increased staining in both the cell body and nucleus compared with control $(F=8.992$ and $F=22.317 ; p<0.01)$, tetanus alone $(F=7.234$ and $F=18.89 ; p<0.01)$, and tetanus plus 5 -HT with anti-sensorin $\mathrm{Ab}(F=7.748$ and $F=19.843 ; p<0.01)$. There was no significant difference in staining in the cell body or nucleus after tetanus plus 5-HT with anti-sensorin Ab compared with control or tetanus alone.

Blocking PKC activity also blocked the release of newly synthesized sensorin and associative LTF (Fig. $6 E-H$ ). Incubation with chelerythrine between 0.5 and $2 \mathrm{~h}$ after tetanus plus 5-HT blocked the decline in sensorin staining (Fig. 6E,F) normally detected by $2 \mathrm{~h}$ after stimulation. Staining for sensorin that increased by 2.5 -fold in both the axons $(244 \pm 17 \%)$ and varicosities $(259 \pm 16 \%)$ at $0.5 \mathrm{~h}$ after tetanus plus 5 -HT $(n=7)$ remained at that high level at $2 \mathrm{~h}$ in both the axons $(235 \pm 16 \%)$ and varicosities $(251 \pm 20 \%)$ when chelerythrine was present between 0.5 and $2 \mathrm{~h}(n=8)$. In the absence of the inhibitor $(n=7)$, sensorin staining declined to control levels in both the axons $(103 \pm 5 \%)$ and varicosities $(101 \pm 6 \%)$. Incubation with chelerythrine between 0.5 and $2 \mathrm{~h}$ also blocked LTF produced by

\footnotetext{
(Figure legend continued.)

inhibitor blocked the decline in sensorin staining $2 \mathrm{~h}$ after tetanus plus 5 -HT. Phase contrast $(\boldsymbol{E}$, top $)$ and immunofluorescent images of sensorin staining ( $\boldsymbol{E}$, bottom) after control, $0.5 \mathrm{~h}$ after tetanus plus $5-\mathrm{HT}, 2 \mathrm{~h}$ after tetanus plus 5 - $\mathrm{HT}$, and $2 \mathrm{~h}$ after tetanus plus 5 -HT with chelerythrine present between 0.5 and $2 \mathrm{~h}$. The rapid increase in sensorin staining in axons (arrows) and varicosities at $0.5 \mathrm{~h}$ does not decline at $2 \mathrm{~h}$ with the PKC inhibitor. Scale bar, $50 \mu \mathrm{m}$. Summary of the changes in sensorin staining after treatments $(\boldsymbol{F})$. Staining in each compartment was normalized to the average staining after control (dashed line at 100\%). ANOVA indicated a significant effect of treatment ( $\mathrm{df}=6$, $48 ; F=42.206 ; p<0.001)$. Compared with control, tetanus plus $5-\mathrm{HT}$ at $0.5 \mathrm{~h}$ produced a significant increase in sensorin staining in the sensory neuron cell bodies $(F=3.951 ; p<0.05)$ and axons and varicosities $(F=18.05$ and $F=18.986 ; p<0.01)$. Without PKC inhibitor, sensorin staining in all compartments at $2 \mathrm{~h}$ after tetanus plus 5 -HT was not significantly different from control. Sensorin staining at $2 \mathrm{~h}$ after tetanus plus 5 -HT with chelerythrine was not significantly different from the staining at $0.5 \mathrm{~h}$ after tetanus plus $5-\mathrm{HT}$, remained significantly higher than control both in the axons and varicosities ( $F=16.071$ and $F=18.052 ; p<0.01$ ), and was significantly higher than the staining $2 \mathrm{~h}$ after tetanus plus 5 -HT without drug in both the axons and varicosities $(F=17.563$ and $F=19.396 ; p<$ 0.01). G, H, PKC inhibition between 0.5 and $2 \mathrm{~h}$ after tetanus plus 5 -HT blocked associative LTF. EPSPs were recorded before (Pre) and $24 \mathrm{~h}$ after (Post) control, 90 min of chelerythrine, tetanus plus 5-HT, and tetanus plus 5-HT with chelerythrine added between 0.5 and $2 \mathrm{~h}$ after paired stimuli (G). Calibration: $20 \mathrm{mV}, 25 \mathrm{~ms}$. Summary of the changes in EPSP amplitudes $24 \mathrm{~h}$ after treatment $(\boldsymbol{H})$. An ANOVA indicated a significant effect of treatment ( $\mathrm{df}=3,22 ; F=$ $125.062 ; p<0.001)$. Change in EPSP amplitude after tetanus plus $5-\mathrm{HT}$ with chelerythrine was not significantly different from control or treatment with chelerythrine alone but was significantly lower than the increase produced by tetanus plus 5 -HT without drug $(F=18.471 ; p<0.01)$. Tetanus plus 5 -HT significantly increased EPSP amplitude compared with control $(F=15.48 ; p<0.01)$.
}

tetanus plus 5-HT (Fig. $6 G, H$ ). The increase in the EPSP amplitudes $24 \mathrm{~h}$ after tetanus plus 5 -HT $(146 \pm 7 \% ; n=6)$ was reduced to control levels (101 $\pm 3 \%)$ when chelerythrine was added between 0.5 and $2 \mathrm{~h}$ after tetanus plus 5-HT $(n=8)$. Thus, PKC activity is also required for the release of the newly synthesized sensorin and associative LTF.

Does PKC activity also contribute to the release of newly synthesized sensorin after five applications of 5-HT? Blocking PKC activity with chelyrethrine during the period of release $0-2 \mathrm{~h}$ after five applications of 5-HT also blocked the decline in sensorin staining normally produced in the absence of the drug. In the presence of chelerythrine $(n=8)$, sensorin staining remained nearly 2.5 -fold higher in both the axons and varicosities $(242 \pm 16$ and $246 \pm 12 \%)$ compared with the decline to control levels in both the axons and varicosities (105 \pm 5 and $108 \pm 5 \%) 2 \mathrm{~h}$ after five applications of 5-HT in the absence of chelerythrine $(n=6)$. The presence of chelerythrine during the $2 \mathrm{~h}$ period after five applications of 5-HT $(n=8)$ also blocked nonassociative LTF. EPSP amplitudes at $24 \mathrm{~h}$ changed $105 \pm 3 \%$ compared with $101 \pm 3 \%$ for control $(n=6)$ and $151 \pm 4 \%$ for five applications of 5-HT in the active of drug $(n=7)$. Thus, PKC activity after stimulation applications of 5-HT and nonassociative LTF. PKC activity after five applications of $5-\mathrm{HT}$, but not during the applications, is Newly released sensorin after tetanus plus 5-HT activates and translocates MAPK

Phosphorylation (activation) and translocation of p42/44 MAPK into the nuclei of sensory neurons is critical for nonassociative LTF (Martin et al., 1997; Purcell et al., 2003; Hu et al., 2004a, 2006; Sharma et al., 2006). The release of the newly synthesized sensorin after five applications of 5-HT binds to autoreceptors, leading to the activation and translocation of the MAPK ( $\mathrm{Hu}$ et al., 2004a, 2006). We used immunocytochemistry to measure the expression and distribution of both phosphorylated p42/44 MAPK and total p42/44 MAPK in sensory neurons to determine whether the release of the newly synthesized sensorin after tetanus plus 5-HT leads to p42/44 MAPK phosphorylation and translocation into the nuclei of sensory neurons $1 \mathrm{~h}$ after stimulation.

The staining for phospho-p42/44 MAPK that increased significantly in both the cell body and nucleus of sensory neurons $1 \mathrm{~h}$ after tetanus plus 5-HT was blocked when the cultures were incubated after stimulation with anti-sensorin Ab (Fig. 7). Compared with controls, staining in the cell body increased nearly twofold and in the nucleus increased by more than threefold after tetanus plus 5-HT. In contrast, the presence of anti-sensorin Ab after tetanus plus 5 -HT blocked the increases in 
staining in both the cell body and the nucleus. Tetanus alone, which fails to evoke the secretion of the newly synthesized sensorin (Fig. 1), did not affect staining for phospho-p42/44 MAPK either in the cell body or nucleus. As was found after five applications of 5-HT (Hu et al., 2004a, 2006), the increase in the nucleus of phospho-p42/44 MAPK is mediated by translocation. Compared with controls (normalized to $100 \%$ in each compartment), staining for total p42/44 MAPK in the nucleus increased to $158 \pm 8 \%$ at $1 \mathrm{~h}$ after tetanus plus 5 -HT $(n=4)$. The increase in the nucleus was accompanied by a compensatory decrease to $71 \pm 4 \%$ in the MAPK staining in the cytoplasm of the sensory neuron cell body. Incubation with anti-sensorin $\mathrm{Ab}(n=4)$ blocked the change in the distribution of total p42/44 MAPK produced by tetanus plus 5-HT (normalized to control at 100\% in each compartment, $101 \pm 6 \%$ in the cytoplasm, and $98 \pm 4 \%$ in the nucleus). Total staining for p42/44 MAPK in the cell body was unaffected by stimulation or incubation with anti-sensorin Ab. Thus, the secretion of the newly synthesized sensorin from the sensory neurons after tetanus plus 5-HT evokes the autocrine activation and translocation of phospho-p42/44 MAPK that is critical for LTF.

\section{Discussion}

Our results indicate that different stimuli activating both unique and common signaling pathways converge in regulating the synthesis and secretion of a neuropeptide whose downstream actions are critical for LTF at Aplysia sensory neurons synapses. With nonassociative LTF (five applications of 5-HT), PI3K, but not $\mathrm{PKC}$, activity is required for the rapid, local increase in sensorin synthesis (Hu et al., 2006). In contrast, with associative LTF (tetanus plus 5-HT), PKC, but not PI3K, activity is required for the rapid, local increase in sensorin synthesis. The increase in sensorin synthesis is rapamycin sensitive. The increase in sensorin synthesis is then followed by the same sequence of events for both nonassociative (Hu et al., 2006) and associative forms of LTF: PKA- and PKC-dependent increases in sensorin secretion and sensorin-dependent activation and translocation of p42/44 MAPK into sensory neuron nuclei. Secretion of newly synthesized sensorin is required primarily for LTF and not short-term facilitation (Hu et al., 2004a) or ITF. The timely and sequential activation of the different signaling pathways mediated in part by the secretion of newly synthesized sensorin are required for LTF that is accompanied by structural plasticity (Glanzman et al., 1990; Sun and Schacher, 1998; Hu et al., 2004a).

\section{$\mathrm{PKC}$ and rapamycin regulate the local synthesis of sensorin after tetanus plus 5-HT}

A single pairing produced a rapid increase in sensorin translation from sensorin mRNA that is located in the cell body, axons, and the distal varicosities (Schacher et al., 1999; Hu et al., 2002; Lyles et al., 2006). Tetanus plus 5-HT, however, modestly increased sensorin in the cell body of the sensory neurons. Overall, the increase in the cell body, when sensorin levels in other compartments were at their maximums, was $\sim 33 \%$ compared with increases in sensorin of $\sim 90 \%$ in the sensory neuron cell bodies after five applications of 5-HT (Fig. 3C) (Hu et al., 2006). In addition, sensorin in the cell body returned to control levels by $2 \mathrm{~h}$ after tetanus plus 5 -HT but remained significantly higher than control levels at $2 \mathrm{~h}$ after five applications of 5-HT (Hu et al., 2006). The quantitative differences could arise from the number of stimuli (five compared with a single pairing) and the amplitude and time course of regulating sensorin synthesis in the cell body by the two signaling pathways (PI3K compared with PKC).
When PKC is activated by phorbol, sensorin levels also increased modestly $(\sim 45 \%)$ in the cell body. The quantitative differences in regulating sensorin synthesis in the cell body may lie in the efficacy of the two signaling pathways in regulating protein synthesis.

Because 5-HT activates PKC, it was surprising that PKC did not play any role in regulating sensorin synthesis with five applications of 5-HT. However, Aplysia sensory neurons express several PKC isoforms (Kruger et al., 1991; Sossin, 2007). Recent studies demonstrated that 5-HT activates the calciumindependent PKC (Apl-II) in sensory neurons, whereas activity plus 5-HT activates the calcium-dependent PKC (Apl-I) (Manseau et al., 2001; Zhao et al., 2006) that is required to produce associative ITF at this synapse. Thus, differences in the activation of specific isoforms of PKC by the two types of stimulation (Apl-II by five applications of 5-HT compared with Apl-I by tetanus plus 5-HT) might explain why PKC regulates sensorin synthesis only after a single strong tetanus ( $20 \mathrm{~Hz}$ for $2 \mathrm{~s}$ ), a single strong tetanus plus 5-HT, multiple tetani in the presence of 5-HT (Zhao et al., 2006), or maximum activation by PDBu. PI3K inhibitor failed to reduce synthesis of sensorin after tetanus plus 5 -HT is likely the result of the absence of a significant activation of this pathway with a single application of 5-HT or a single pairing of tetanus plus 5-HT. Increased phosphorylation of a PI3K substrate, Akt/protein kinase B, was observed in isolated ganglia after five applications of 5-HT, whereas one application of 5-HT did not significantly increase phosphorylation compared with controls (J. Liu, J.-Y. Hu, J. H. Schwartz, and S. Schacher, unpublished observations). Thus, the activation of a specific isoform of PKC might influence sensorin synthesis after tetanus plus 5-HT, whereas sufficient activation of PI3K by repeated applications of 5-HT (Udo et al., 2005) may regulate sensorin synthesis after five applications of 5-HT. Either signaling pathway could then lead to the regulation of rapamycin-sensitive protein synthesis (Khan et al., 2001; Pepio et al., 2002) required for increasing sensorin levels and other proteins at distal varicosities even without the sensory neuron cell body. Thus, the machinery for local protein synthesis and its various regulatory pathways (PKC or PI3K dependent and rapamycin sensitive) are distributed in the cell body, axons, and distal varicosities.

Although isolated sensory neurons contain autoreceptors capable of responding to exogenous sensorin (Hu et al., 2004a), sensorin synthesis in sensory neurons did not increase after tetanus plus 5-HT when the motor neuron L7 was absent. However, bypassing stimulus-dependent activation of receptors with direct activation of PKC by phorbol led to a smaller but significant increase in sensorin expression throughout the sensory neuron when L7 was absent. This increase parallels that found by Zhao et al. (2006) who found that strong paired stimulation, repeated tetani applied to isolated sensory neurons in the presence of 5-HT, also activated type 1 PKC (Zhao et al., 2006). The absence of a significant change in sensorin expression in isolated sensory neurons with a single pairing or the smaller increase with PDBu compared with cocultures could arise from a reduction in sensorin mRNA levels at distal sites (Hu et al., 2002; Lyles et al., 2006) and a reduction in the levels of the 5-HT receptors at distal sites that lead to the activation of the appropriate $\mathrm{PKC}$ isoform after a single tetanus plus 5-HT (Sun and Schacher, 1996, 1998; Hu et al., $2002,2004 b)$. In addition, activity plus 5 -HT might regulate a retrograde signal from the target cell L7 that contributes to the changes in sensorin expression in the sensory neuron. In the absence of the motor cell, this missing signal could prevent the changes in sensorin synthesis produced by tetanus plus 5-HT. 


\section{PKA and PKC regulate sensorin secretion that leads to the activation and translocation of p42/44 MAPK and LTF}

Both PKA and PKC activities contribute to sensorin secretion after five applications of 5-HT (Hu et al., 2006) or tetanus plus 5-HT. Type II PKA is enriched at synapses of Aplysia and is maintained close to the plasma membrane by interaction between the regulatory subunit RII and membrane anchoring proteins (AKAPs) (Liu et al., 2004). This interaction is required for the release of sensorin (Hu et al., 2006). The persistent activation of type II PKA by cAMP by either five applications of 5-HT (Bernier et al., 1982; Greenberg et al., 1987; Muller and Carew, 1998; Liu et al., 2004) or pairing tetanus plus 5-HT (Abrams et al., 1991, 1998) could phosphorylate key proteins that enhance the secretion of the neuropeptide (Goodman et al., 1996; Han et al., 1999; Jovanovic et al., 2000; Angers et al., 2002). Persistent activation of PKC at synaptic terminals of sensory neurons (Sossin et al., 1994; Sutton et al., 2000, 2004; Zhao et al., 2006) may also lead to the phosphorylation of synaptic proteins necessary for secretion of neuropeptides (Nakhost et al., 2003; Houeland et al., 2007; Sieburth et al., 2007) or the phosphorylation of cytoskeletal proteins or other substrates that regulate the recruitment of neuropeptide-containing vesicles to appropriate positions near the plasma membrane for subsequent release (Knox et al., 1992; Nakhost et al., 1998, 2002; White et al., 1998; Smith, 1999; Nagy et al., 2002; Gruenbaum et al., 2003). Inhibition of either kinase immediately after stimulation blocks both release and LTF. Thus, persistent activation of the two kinases are likely to act cooperatively by either phosphorylating different sets of synaptic proteins with complementary and essential functions and/or different sites on the same proteins that regulate neuropeptide release. Because different PKC isoforms appear to be activated by five applications of 5-HT and tetanus plus 5-HT, it is unclear which isoform of $\mathrm{PKC}$ is regulating neuropeptide secretion during the period after stimulation. Persistent activation of an atypical PKC isoform recently identified in Aplysia (Sossin, 2007) after tetanus plus 5-HT or five applications of 5-HT may function in regulating secretion in a manner analogous to the persistent activation of an atypical PKC important for maintaining long-term potentiation (LTP) in hippocampus (Ling et al., 2002; Serrano et al., 2005). Thus, the persistent activation of both PKA and PKC lead to the release of the newly synthesized sensorin for different forms of LTF. Because sensorin release can occur without the sensory neuron cell body, the distal processes and varicosities must contain the appropriate apparatus for packaging and secreting this locally synthesized neuropeptide to activate other signaling pathways critical for LTF. Our results for sensorin parallel the stimulus-induced regulation of local synthesis and secretion of BDNF that are important for long-lasting forms of LTP in the hippocampus (Hall et al., 2000; Pang et al., 2004).

One such pathway activated by sensorin (and BDNF) is the p42/44 MAPK pathway, whose phosphorylated form is translocated into the nucleus of the sensory neuron. This autocrine action of sensorin is critical for the expression of both nonassociative LTF and associative LTF (Hu et al., 2004a, 2006). The activation of PKA in the cell body by $5-\mathrm{HT}$ also leads to the translocation of PKA into the nucleus (Bacskai et al., 1993) and may facilitate the translocation of MAPK. PKA activation by 5-HT is a prerequisite for the activation and translocation of MAPK by sensorin (Hu et al., 2004a, 2006). The translocated MAPK and PKA phosphorylate transcription factors and other components within the nucleus to regulate gene expression required for LTF. Thus, long-lasting change in behaviorally relevant synapses is orchestrated by the timely and persistent activa- tion of signaling pathways first by the initial stimuli and then followed by the actions of a secreted neuropeptide.

\section{References}

Abrams TW, Karl KA, Kandel ER (1991) Biochemical studies of stimulus convergence during classical conditioning in Aplysia: dual regulation of adenylate cyclase by calcium/calmodulin and transmitter. J Neurosci 11:2655-2665.

Abrams TW, Yovell Y, Onyike CU, Cohen JE, Jarrard HE (1998) Analysis of sequence-dependent interactions between transient calcium and transmitter stimuli in activating adenylyl cyclase in Aplysia: possible contribution to CS-US sequence requirement during conditioning. Learn Mem 4:496-509.

Alberini CM, Ghirardi M, Metz R, Kandel ER (1994) C/EBP is an immediated-early gene required for the consolidation of long-term facilitation in Aplysia. Cell 76:1099-1114.

Angers S, Fioravante D, Chin J, Cleary LJ, Bean AJ, Byrne JH (2002) Serotonin stimulates phosphorylation of Aplysia synapsin and alters its subcellular distribution. J Neurosci 22:5412-5422.

Bacskai BJ, Hochner B, Mahaut-Smith M, Adams SR, Kaang BK, Kandel ER, Tsien RY (1993) Spatially resolved dynamics of cAMP and protein kinase A subunits in Aplysia sensory neurons. Science 260:222-226.

Bailey CH, Chen M (1983) Morphological basis of long-term habituation and sensitization. Science 220:91-93.

Bailey CH, Chen M (1988) Long-term memory in Aplysia modulates the total number of varicosities of single identified sensory neurons. Proc Natl Acad Sci USA 85:2373-2377.

Bailey CH, Chen M (1989) Time course of structural changes at identified sensory neuron synapses during long-term sensitization in Aplysia. J Neurosci 9:1774-1780.

Bailey CH, Chen M, Montarolo PG, Kandel ER, Schacher S (1992) Inhibitors of protein and RNA synthesis block structural and functional changes accompanying long-term synaptic facilitation and inhibition in Aplysia sensory neurons. Neuron 9:749-758.

Bailey CH, Kaang BK, Chen M, Martin KC, Lim CS, Casadio A, Kandel ER (1997) Mutation in the phosphorylation sites of MAP kinase blocks learning-related internalization of apCAM in Aplysia sensory neurons. Neuron 18:913-924.

Bailey CH, Giustetto M, Zhu H, Chen M, Kandel ER (2000) A novel function for serotonin-mediated short-term facilitation in Aplysia: conversion of a transient, cell-wide homosynaptic hebbian plasticity into a persistent, protein synthesis-independent synapse-specific enhancement, Proc Natl Acad Sci USA 97:11581-11586.

Bartsch D, Casadio A, Karl KA, Serodio P, Kandel ER (1998) CREB1 encodes a nuclear activator, a repressor, and a cytoplasmic modulator that form a regulatory unit critical for long-term facilitation. Cell 95:211-223.

Bernier L, Castellucci VF, Kandel ER, Schwartz JH (1982) Facilitatory transmitter causes a selective and prolonged increase in adenosine $3^{\prime}: 5^{\prime}$ monophosphate in sensory neurons mediating the gill and siphon withdrawal reflex in Aplysia. J Neurosci 2:1682-1691.

Buonomano DV, Byrne JH (1990) Long-term synaptic changes produced by a cellular analog of classical conditioning in Aplysia. Science 249:420-423.

Dash P, Hochner B, Kandel ER (1990) Injection of cAMP responsive element into the nucleus of Aplysia sensory neurons block s long-term facilitation. Nature 345:718-721.

Eliot LS, Hawkins RD, Kandel ER, Schacher S (1994) Pairing-specific, activity-dependent presynaptic facilitation at Aplysia sensory-motor neuron synapses in isolated cell culture. J Neurosci 14:368-383.

Glanzman DL, Kandel ER, Schacher S (1990) Target-dependent structural changes accompany long-term synaptic facilitation in Aplysia neurons. Science 249:799-802.

Goodman LJ, Valverde J, Lim F, Geschwind MD, Federoff HJ, Geller AI, Hefti F (1996) Regulated release and polarized localization of brain-derived neurotrophic factor in hippocampal neurons. Mol Cell Neurosci 7:222-238.

Greenberg SM, Castellucci VF, Bayley H, Schwartz JH (1987) A molecular mechanism for long-term sensitization in Aplysia. Nature 329:62-65.

Gruenbaum LM, Gilligan DM, Picciotto MR, Marinesco S, Carew TJ (2003) Identification and characterization of Aplysia adducing, an Aplysia cytoskeletal protein homologous to mammalian adducins: increased phos- 
phorylation at a protein kinase $\mathrm{C}$ consensus site during long-term synaptic facilitation. J Neurosci 23:2675-2685.

Hall J, Thomas KL, Everitt BJ (2000) Rapid and selective induction of BDNF expression in the hippocampus during contextual learning. Nat Neurosci 3:533-535.

Han W, Ng YK, Axelrod D, Levitan ES (1999) Neuropeptide release by efficient recruitment of diffusing cytoplasmic secretory vesicles. Proc Natl Acad Sci USA 96:14577-14582.

Houeland G, Nakhost A, Sossin WS, Castellucci VF (2007) PKC modulation of transmitter release by SNAP-25 at sensory-to-motor synapses in Aplysia. J Neurophysiol 97:134-143.

Hu JY, Meng X, Schacher S (2002) Target interaction regulates distribution and stability of specific mRNAs. J Neurosci 22:2669-2678.

Hu JY, Meng X, Schacher S (2003) Redistribution of syntaxin mRNA in neuronal cell bodies regulates protein expression and transport during synapse formation and long-term synaptic plasticity. J Neurosci 23:18041815.

Hu JY, Glickman L, Wu F, Schacher S (2004a) Serotonin regulates the secretion and autocrine action of a neuropeptide to activate MAPK required for long-term tacilitation in Aplysia. Neuron 43:373-385.

Hu JY, Goldman J, Wu F, Schacher S (2004b) Target-dependent release of a presynaptic neuropeptide regulates the formation and maturation of specific synapses in Aplysia. J Neurosci 24:9933-9943.

Hu JY, Wu F, Schacher S (2006) Two signaling pathways regulate the expression and secretion of a neuropeptide required for long-term facilitation in Aplysia. J Neurosci 26:1026-1035.

Jovanovic JN, Czernik AJ, Fienberg AA, Greengard P, Sihra TS (2000) Synapsins as mediators of BDNF-enhanced neurotransmitter release. Nat Neurosci 3:323-329.

Kandel ER (2001) The molecular biology of memory storage: a dialogue between genes and synapses. Science 294:1030-1038.

Khan A, Pepio AM, Sossin WS (2001) Serotonin activates S6 kinase in a rapamycin-sensitive manner in Aplysia synaptosomes. J Neurosci 21:382-391.

Kim JH, Udo H, Li HL, Youn TY, Chen M, Kandel ER, Bailey CH (2003) Presynaptic activation of silent synapses and growth of new synapses contribute to intermediate and long-term facilitation in Aplysia. Neuron 40:151-165.

Knox RJ, Quattrocki EA, Connor JA, Kaczmarek LK (1992) Recruitment of $\mathrm{Ca}^{2+}$ channels by protein kinase $\mathrm{C}$ during rapid formation of putative neuropeptide release sites in isolated Aplysia neurons. Neuron 8:883-889.

Kruger KE, Sossin WS, Sacktor TC, Bergold PJ, Beushausen S, Schwartz JH (1991) Cloning and characterization of $\mathrm{Ca}^{2+}$-dependent and $\mathrm{Ca}^{2+}$ independent PKCs expressed in Aplysia sensory cells. J Neurosci 11:23032313.

Lin XY, Glanzman DL (1994) Hebbian induction of long-term potentiation of Aplysia sensorimotor synapses: partial requirement for activation of a NMDA-related receptor. Proc R Soc Lond B Biol Sci 255:215-221.

Ling DS, Benardo LS, Serrano PA, Blace N, Kelly MT, Crary JF, Sacktor TC (2002) Protein kinase Mzeta is necessary and sufficient for LTP maintenance. Nat Neurosci 5:295-296.

Liu J, Hu JY, Schacher S, Schwartz JH (2004) The two regulatory subunits of Aplysia protein kinase A mediate distinct functions in producing synaptic plasticity. J Neurosci 24:2465-2474.

Liu K, Hu JY, Wang D, Schacher S (2003) Protein synthesis at synapse versus cell body: enhanced but transient expression of long-term facilitation at isolated synapses. J Neurobiol 56:275-286.

Lyles V, Zhao Y, Martin KC (2006) Synapse formation and mRNA localization in cultured Aplysia neurons. Neuron 49:349-356.

Manseau F, Fan X, Hueftlein T, Sossin WS, Castellucci VF (2001) $\mathrm{Ca}^{2+}$ independent protein kinase C Apl II mediates the serotonin-induced facilitation at depressed Aplysia sensorimotor synapses. J Neurosci 21:1247-1256.

Martin KC, Michael D, Rose JC, Barad M, Casadio A, Zhu H, Kandel ER (1997) MAP kinase translocates into the nucelus of the presynaptic cell and is required for long-term facilitation in Aplysia. Neuron 18:899-912.

Michael D, Martin KC, Seger R, Ning MM, Baston R, Kandel ER (1998) Repeated pulses of serotonin required for long-term facilitation activate mitogen-activated protein kinase in sensory neurons of Aplysia. Proc Natl Acad Sci USA 95:1864-1869.

Montarolo PG, Goelet P, Castellucci VF, Morgan J, Kandel ER, Schacher S
(1986) A critical period for macromolecular synthesis in long-term heterosynaptic facilitation in Aplysia. Science 234:1249-1254.

Muller U, Carew TJ (1998) Serotonin induces temporally and mechanistically distinct phases of persistent PKA activity in Aplysia sensory neurons. Neuron 21:1423-1434.

Murphy GG, Glanzman DL (1997) Mediation of classical conditioning in Aplysia californica by long-term potentiation of sensorimotor synapses. Science 278:467-471.

Nagy G, Matti U, Nehring RB, Binz T, Rettig J, Neher E, Sorensen JB (2002) Protein kinase C-dependent phosphorylation of synaptosome-associated protein of $25 \mathrm{kDa}$ at Ser187 potentiates vesicle recruitment. J Neurosci 22:9278-9286.

Nakhost A, Forscher P, Sossin WS (1998) Binding of protein kinase C isoforms to actin in Aplysia. J Neurochem 71:1221-1231.

Nakhost A, Kabir N, Forscher P, Sossin WS (2002) Protein kinase C isoforms are translocated to microtubules in neurons. J Biol Chem 277:40633-40639.

Nakhost A, Houeland G, Castellucci VF, Sossin WS (2003) Differential regulation of transmitter release by alternatively spliced forms of synaptotagmin I. J Neurosci 23:6238-6244.

Nazif FA, Byrne JH, Cleary LJ (1991) cAMP induces long-term morphological changes in sensory neurons of Aplysia. Brain Res 539:324-327.

Occor KA, Walters ET, Byrne JH (1985) Associative conditioning analog selectively increases cAMP levels of tail sensory neurons in Aplysia. Proc Natl Acad Sci USA 82:2548-2552.

Ormond J, Hislop J, Zhao Y, Webb N, Vaillaincourt F, Dyer JR, Ferraro G, Barker P, Martin KC, Sossin WS (2004) ApTrkl, a Trk-like receptor, mediates serotonin-dependent ERK activation and long-term facilitation in Aplysia sensory neurons. Neuron 44:715-728.

Pang PT, Lu B (2004) Regulation of late-phase LTP and long-term memory in normal and aging hippocampus: role of secreted proteins tPA and BDNF. Ageing Res Rev 3:407-430.

Pepio AM, Thibault GL, Sossin WS (2002) Phosphoinositide-dependent kinase phosphorylation of protein kinase C Apl II increases during intermediate facilitation in Aplysia. J Biol Chem 277:37116-37123.

Purcell AL, Sharma SK, Bagnall MW, Sutton MA, Carew TJ (2003) Activation of a tyrosine kinase-MAPK cascade enhances the induction of longterm synaptic facilitation and long-term memory in Aplysia. Neuron 37:473-484.

Rayport SG, Schacher S (1986) Synaptic plasticity in vitro: cell culture of identified Aplysia neurons mediating short-term habituation and sensitization. J Neurosci 6:759-763.

Roberts AC, Glanzman DL (2003) Learning in Aplysia: looking at synaptic plasticity from both sides. Trends Neurosci 26:662-670.

Sacktor TC, Schwartz JH (1990) Sensitizing stimuli cause translocation of protein kinase C in Aplysia sensory neurons. Proc Natl Acad Sci USA 87:2036-2039.

Schacher S, Montarolo PG, Kandel ER (1990) Selective short- and longterm effects of serotonin, small cardioactive peptide and tetanic stimulation on sensorimotor synapses of Aplysia in culture. J Neurosci 10:3286-3294.

Schacher S, Montarolo PG, Kandel ER (1993) cAMP and arachidonic acid simulate long-term structural and functional changes produced by neurotransmitters in Aplysia sensory neurons. Neuron 10:1079-1088.

Schacher S, Wu F, Sun ZY (1997) Pathway-specific synaptic plasticity: activity-dependent enhancement and suppression of long-term heterosynaptic facilitation at converging inputs on a single target. J Neurosci 17:597-606.

Schacher S, Wu F, Panyko JD, Sun ZY, Wang D (1999) Expression and branch-specific export of mRNA are regulated by synapse formation and interaction with specific postsynaptic targets. J Neurosci 19:6338-6347.

Schuster MJ, Camardo JS, Siegelbaum SA, Kandel ER (1985) Cyclic-AMPdependent protein kinase closes the serotonin-sensitive $\mathrm{K}^{+}$channels of Aplysia sensory neurons in cell-free membrane patches. Nature 313:392-395.

Serrano P, Yao Y, Sacktor TC (2005) Persistent phosphorylation of protein kinase Mzeta maintains late-phase long-term potentiation. J Neurosci 25:1979-1984.

Sharma SK, Sherff CM, Stough S, Hsuan V, Carew TJ (2006) A tropomyosin-related kinase B ligand is required for ERK activation, longterm synaptic facilitation and long-term memory in Aplysia. Proc Natl Acad Sci USA 103:14206-14210. 
Sieburth D, Madison JM, Kaplan JM (2007) PKC-1 regulates secretion of neuropeptides. Nat Neurosci 10:49-57.

Smith C (1999) A persistent activity-dependent facilitation in chromaffin cells is caused by $\mathrm{Ca}^{2+}$ activation of protein kinase C. J Neurosci 19:589-598.

Sossin WS (2007) Isoform specificity of protein kinase Cs in synaptic plasticity. Learn Mem 14:236-246.

Sossin WS, Schwartz JH (1992) Selective activation of calcium-activated PKCs in Aplysia neurons by 5-HT. J Neurosci 9:3218-3229.

Sossin WS, Sacktor TC, Schwartz JH (1994) Persistent activation of protein kinase C during the development of long-term facilitation in Aplysia. Learn Mem 1:189-202.

Sugita S, Goldsmith JR, Baxter DA, Byrne JH (1992) Involvement of protein kinase $\mathrm{C}$ in serotonin-induced spike broadening and synaptic facilitation of sensorimotor connections in Aplysia. J Neurophysiol 68:643-651.

Sugita S, Baxter DA, Byrne JH (1997) Modulation of a cAMP/protein kinase A cascade by protein kinase C in sensory neurons of Aplysia. J Neurosci 17:7237-7244.

Sun ZY, Schacher S (1996) Development of short-term heterosynaptic facilitation at Aplysia sensorimotor synapses is accompanied by changes in the functional expression of presynaptic serotonin receptors. J Neurophysiol 76:2250-2261.

Sun ZY, Schacher S (1998) Binding of serotonin to receptors at multiple sites is required for structural plasticity accompanying long-term facilitation of Aplysia sensorimotor synapses. J Neurosci 18:3991-4000.

Sutton MA, Carew TJ (2000) Parallel molecular pathways mediate expression of distinct forms of intermediate-term facilitation at tail sensorymotor synapses in Aplysia. Neuron 26:219-231.
Sutton MA, Bagnall MW, Sharma SK, Shobe J, Carew TJ (2004) Intermediate-term memory for site-specific sensitization in Aplysia is maintained by persistent activation of protein kinase C. J Neurosci 24:3600-3609.

Sweatt JD, Kandel ER (1989) Persistent and transcription-dependent in crease in protein phosphorylation in long-term facilitation of Aplysia sensory neurons. Nature 339:51-54.

Udo H, Jin I, Kim JH, Li HL, Youn T, Hawkins RD, Kandel ER, Bailey CH (2005) Serotonin-induced regulation of the actin network for learningrelated synaptic growth requires Cdc42, N-WASP, and PAK in Aplysia sensory neurons. Neuron: 45:887-901.

White BH, Nick TA, Carew TJ, Kaczmarek LM (1998) Protein kinase C regulates a vesicular class of calcium channels in the bag cell neurons of Aplysia. J Neurophysiol 80:2514-2520.

Wu F, Friedman L, Schacher S (1995) Transient versus persistent functional and structural changes associated with facilitation of Aplysia sensorymotor synapses are second messenger dependent. J Neurosci 15:7517-7527.

Yamamoto N, Hegde AN, Chain DG, Schwartz JH (1999) Activation and degradation of the transcription factor C/EBP during long-term facilitation in Aplysia. J Neurochem 73:2415-2423.

Zhao Y, Leal K, Abi-Farah C Martin KC, Sossin WS, Klein M (2006) Isoform specificity of PKC translocation in living Aplysia sensory neurons and a role for $\mathrm{Ca}^{2+}$-dependent PKC APL I in the induction of intermediateterm facilitation. J Neurosci 26:8847-8856.

Zhu H, Wu F, Schacher S (1997) Site-specific and sensory neurondependent increases in postsynaptic glutamate sensitivity accompany serotonin-induced long-term facilitation at Aplysia sensorimotor synapses. J Neurosci 17:4976-4986. 